\title{
Constitutive relations and Schrödinger's formulation of nonlinear electromagnetic theories
}

\author{
Paolo Aschieri ${ }^{a, b}$ and Sergio Ferrara ${ }^{c, d}$ \\ ${ }^{a}$ Dipartimento di Scienze e Tecnologie Avanzate, Università del Piemonte Orientale, \\ Viale T. Michel 11, 15121 Alessandria, Italy \\ ${ }^{b}$ INFN, Sezione di Torino, gruppo collegato di Alessandria, \\ Viale T. Michel 11, 15121 Alessandria, Italy \\ ${ }^{c}$ Physics Department, Theory Unit, CERN, \\ CH 1211, Geneva 23, Switzerland \\ ${ }^{d}$ INFN - Laboratori Nazionali di Frascati, \\ Via Enrico Fermi 40, I-00044 Frascati, Italy \\ E-mail: aschieri@to.infn.it, sergio.ferrara@cern.ch
}

ABSTRACT: We present a systematic study of nonlinear and higher derivatives extensions of electromagnetism. We clarify when action functionals $S[F]$ can be explicitly obtained from arbitrary (not necessarily self-dual) nonlinear equations of motion. We show that the "Deformed twisted self-duality condition" proposal originated in the context of supergravity counterterms is actually the general framework needed to discuss self-dual theories starting from a variational principle.

We generalize to nonlinear and higher derivatives theories Schrödinger formulation of Born-Infeld theory, and for the latter, and more in general for nonlinear theories, we derive a closed form expression of the corresponding deformed twisted self-duality conditions. This implies that the hypergeometric expression entering these duality conditions and leading to Born-Infeld theory satisfies a hidden quartic equation.

Keywords: Duality in Gauge Field Theories, Global Symmetries

ARXIV EPRINT: 1302.4737 


\section{Contents}

1 Introduction 1

$2 \mathrm{U}(1)$ duality rotations in nonlinear and higher derivatives electromagnetism

2.1 Action functionals from equations of motion 3

$2.2 \mathrm{U}(1)$ duality rotations $\quad 6$

2.3 Complex and chiral variables 8

2.4 The action functional $\mathcal{I}\left[T^{-}, \overline{T^{-}}\right] \quad 9$

2.5 From $S[F]$ to $\mathcal{I}\left[T^{-}, \overline{T^{-}}\right]$via Legendre transform 11

3 Constitutive relations without self-duality $\quad \mathbf{1 2}$

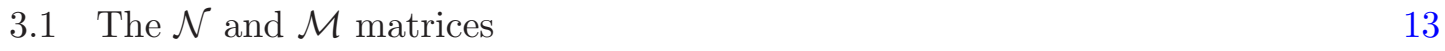

$\begin{array}{lll}3.2 & \text { Complex variables } & 15\end{array}$

4 Schrödinger approach to self-duality conditions $\quad 16$

$\begin{array}{ll}4.1 \text { Chiral variables } & 18\end{array}$

$\begin{array}{lll}5 & \text { Nonlinear theories without higher derivatives } & 19\end{array}$

$\begin{array}{lll}5.1 & \text { Born-Infeld nonlinear theory } 20\end{array}$

5.2 The hypergeometric function and its hidden identity 20

$\begin{array}{ll}5.3 \text { General nonlinear theory } & 21\end{array}$

A Examples of higher derivatives theories $\quad 22$

B The action functional $S[F]$ from $\mathcal{I}\left[T^{-}, \overline{T^{-}}\right] \quad 23$

C The energy momentum tensor and its trace $\quad 24$

\section{Introduction}

Duality is a leading paradigm of theoretical physics. Electric-magnetic duality is one of the oldest and most studied examples. Maxwell theory is self-dual, i.e., admits duality symmetry under rotation of the electric field into the magnetic one. Schrödinger [1] was the first to show that the nonlinear theory of electromagnetism of Born and Infeld, quite remarkably has the same U(1) duality symmetry property. The study of electric-magnetic duality symmetry has found further motivations since its appearance in extended supergravity theories [3-6]. In [4] the first example of a noncompact duality rotation group was considered, it arises in $N=4$ supergravity and is due to scalar fields transforming under duality rotations. These results triggered further investigations in the general structure of self-dual theories. In particular the symplectic formalism for nonlinear electromagnetism coupled to scalar and fermion fields was initiated in [7], there the duality groups were shown to be subgroups of noncompact symplectic groups (the compact case being recovered in the 
absence of scalar fields). A nonlinear example is Born-Infeld electrodynamics coupled to axion and dilaton fields [8]. Another relevant aspect [11] is that the spontaneous breaking of $N=2$ rigid supersymmetry to $N=1$ can lead to a Goldstone vector multiplet whose action is the supersymmetric and self-dual Born-Infeld action [9, 10]. Higher supersymmetric Born-Infeld type actions are also self-dual and related to spontaneous supersymmetry breakings in field theory $[12-15]$ and in string theory $[17,16]$.

Duality symmetry is a powerful tool to investigate the structure of possible counterterms in extended supergravity. After the explicit computations that showed the 3-loop UV finiteness of $N=8$ supergravity [18], an explanation based on $E_{7(7)}$ duality symmetry was provided [19-22]. Furthermore duality symmetry arguments have also been used to suggest all loop finiteness of $N=8$ supergravity [23, 24]. Related to these developments, in [25] a proposal on how to implement duality rotation invariant counterterms in a corrected action $S[F]$ leading to a self-dual theory was put forward under the name of "deformed twisted self-duality conditions" (see eq. (2.32)). Examples included counterterms dependent on derivatives of the field strength. The proposal (renamed "nonlinear twisted self-duality conditions") was further elaborated in [26] and [27]; see also [28], and [29, 30], for the supersymmetric extensions and examples. The proposal is equivalent to a formulation of self-dual theories using auxiliary fields studied in [31] and [32] in case of nonlinear electromagnetism without higher derivatives of the field strength. This coincidence has been brought to light in a very recent paper [33].

The supergravity motivated studies have provided new examples of self-dual theories and have touched upon basic issues like consistency and equivalence of different formulations of self-duality conditions, reconstruction of the action from these conditions and of duality invariant expressions. This paper is a systematic study of these issues.

A nonlinear and higher derivative electromagnetic theory is determined by defining, eventually implicitly, the relation between the electric field strength $F$ (given by the electric field $E$ and the magnetic induction $B$ ) and the magnetic field strength $G$ (given by the magnetic field $H$ and the electric displacement $D$ ). We call constitutive relations the relations defining $G$ in terms of $F$ or vice versa.

We begin section 2 by proving that (locally) the equations of motions of an arbitrary, not necessarily self-dual, nonlinear electromagnetic theory satisfying an integrability condition can always be obtained from a variational principle via an action $S[F]$ that is explicitly computed (reconstructed) from the constitutive relations. This reconstruction procedure works also for theories with higher derivatives if we further assume that they can be obtained from an action principle.

We then study the general theory of U(1) duality rotations. Self-duality of the equations of motion constrains the constitutive relations. The deformed twisted self-duality conditions are just constitutive relations obtained from a variational procedure. In these deformed twisted self-duality conditions the dependence of $G$ from $F$ is given implicitly, but the constraint that leads to self-dual theories is easily implemented. This is due to the use of the complex and chiral variables $T^{+}, T^{-}, \overline{T^{+}}, \overline{T^{-}}$that are the chiral projections of the variables $T=F-i G$ and $\bar{T}=F+i G$ introduced by Schrödinger [1, 34]. The fields $T^{+}, T^{-}, \overline{T^{+}}, \overline{T^{-}}$have definite electric-magnetic duality charge and chirality: $\left(T^{+},+1,+1\right),\left(T^{-},+1,-1\right),\left(\overline{T^{+}},-1,-1\right),\left(\overline{T^{-}},-1,+1\right)$. 
The action $S[F]$ can always be reconstructed from the action $\mathcal{I}\left[\mathcal{T}^{-}, \overline{\mathcal{T}^{-}}\right]$that determines the deformed twisted self-duality conditions, and vice versa. Indeed, as also shown in [32], the two actions are related by a Legendre transformation. This shows that the deformed twisted self-duality conditions are the general framework needed to discuss self-dual theories obtained from a variational principle.

Section 3 is devoted to a detailed study of the constitutive relations of the kind ${ }^{*} G_{\mu \nu}=$ $\mathcal{N}_{2} F_{\mu \nu}+\mathcal{N}_{1}{ }^{*} F_{\mu \nu}$ where $\mathcal{N}_{1}$ and $\mathcal{N}_{2}$ are real (pseudo)scalar functions of $F, G$ and their derivatives. These are not the most general constitutive relations because $\mathcal{N}_{1}$ and $\mathcal{N}_{2}$ are not differential operators and do not act on the $\mu \nu$-indices of $F_{\mu \nu}$ and its Hodge dual ${ }^{*} F_{\mu \nu}$. However they describe a wide class of nonlinear theories. For example theories without higher derivatives are determined by this kind of relations. ${ }^{1}$ Equivalent but more duality symmetric formulations of these constitutive relations are then investigated. In particular we formulate consistent constitutive relations in terms of the complex variables $T=F-i G$ and $\bar{T}=F+i G$, thus generalizing Schrödinger study of Born-Infeld theory [1,34].

In section 4 the constitutive relations of section 3 are constrained to define self-dual theories. These self-dual constitutive relations turn out to be very simple. They are determined for example by expressing the ratio $\frac{T_{\mu \nu} \bar{T}^{\mu \nu}}{\left|T_{\mu \nu} T^{\mu \nu}\right|}$ in terms of $T, \bar{T}$ and their derivatives. In particular we see that self-duality constraints the phases of $T_{\mu \nu}{ }^{*} T^{\mu \nu}$ and $T_{\mu \nu} T^{\mu \nu}$ to differ by a $-\pi / 2$ angle and the square of their moduli to differ by $\left|T_{\mu \nu} \bar{T}^{\mu \nu}\right|^{2}$.

Section 5 considers self-dual theories that do not involve higher derivatives of the field strength. In this case the natural independent variable is $\left|T_{\mu \nu} T^{\mu \nu}\right|$. We present a closed form expression of the deformed twisted self-duality conditions that determine Born-Infeld theory. Comparison of this expression with the one in terms of a hypergeometric function $\mathfrak{F}$ previously considered in [26] leads to a hidden quartic equation for $\mathfrak{F}$. This quartic equation is not just a feature of Born-Infeld theory. It also enters the explicit relation we obtain between deformed twisted self-duality conditions of any nonlinear theory and the corresponding constitutive relations in the Schrödinger's variables $T, \bar{T}$.

In the appendices we provide examples of self-dual theories with higher derivatives, a basic result on the energy momentum tensor of nonlinear theories and details on a technical calculation.

\section{$2 \mathrm{U}(1)$ duality rotations in nonlinear and higher derivatives electromag- netism}

\subsection{Action functionals from equations of motion}

Nonlinear and higher derivatives electromagnetism is described by the equations of motion

$$
\begin{aligned}
& \partial_{\mu} \widetilde{F}^{\mu \nu}=0, \\
& \partial_{\mu} \widetilde{G}^{\mu \nu}=0, \\
& \widetilde{G}^{\mu \nu}=h^{\mu \nu}[F, \lambda] .
\end{aligned}
$$

\footnotetext{
${ }^{1}$ Indeed in this case the elementary antisymmetric 2-tensors in the theory are only $F_{\mu \nu}$ and its Hodge dual ${ }^{*} F_{\mu \nu}$, hence any antisymmetric 2-tensor will be a linear combination (with coefficients dependent on the field strength) of $F_{\mu \nu}$ and ${ }^{*} F_{\mu \nu}$.
} 
The first two simply state that the 2-forms $F$ and $G$ are closed, $d F=d G=0$, indeed $\widetilde{F}^{\mu \nu} \equiv \frac{1}{2} \varepsilon^{\mu \nu \rho \sigma} F_{\rho \sigma}, \widetilde{G}^{\mu \nu} \equiv \frac{1}{2} \varepsilon^{\mu \nu \rho \sigma} G_{\rho \sigma}\left(\right.$ with $\left.\varepsilon^{0123}=1\right)$. The last set $\widetilde{G}^{\mu \nu}=h^{\mu \nu}[F, \lambda]$, where $\lambda$ is the dimensionful parameter typically present in a nonlinear theory, ${ }^{2}$ are the constitutive relations. They specify the dynamics and determine the magnetic field strength $G$ as a functional in term of the electric field strength $F$, and, vice versa, determine $F$ in term of $G$, indeed $F$ and $G$ should be treated on equal footing in (2.1)-(2.3). The square bracket notation $h^{\mu \nu}[F, \lambda]$ stems from the possible dependence of $h^{\mu \nu}$ from derivatives of $F$.

Since in general we consider curved background metrics $g_{\mu \nu}$, it is convenient to introduce the $*$-Hodge operator; on an arbitrary antisymmetric tensor $F_{\mu \nu}$ it is defined by

$$
{ }^{*} F_{\mu \nu}=\frac{1}{2 \sqrt{g}} g_{\mu \alpha} g_{\nu \beta} \varepsilon^{\alpha \beta \rho \sigma} F_{\rho \sigma}=\frac{1}{\sqrt{g}} \widetilde{F}_{\mu \nu}
$$

where $g=-\operatorname{det}\left(g_{\mu \nu}\right)$, and it squares to minus the identity. The constitutive relations $(2.3)$ implicitly include also a dependence on the background metric $g_{\mu \nu}$ and for example in case of usual electromagnetism they read $G_{\mu \nu}={ }^{*} F_{\mu \nu}=\frac{1}{\sqrt{g}} \widetilde{F}_{\mu \nu}$, while for Born-Infeld theory,

$$
S_{B I}=\frac{1}{\lambda} \int d^{4} x \sqrt{g}\left(1-\sqrt{1+\frac{1}{2} \lambda F^{2}-\frac{1}{16} \lambda^{2}\left(F^{*} F\right)^{2}}\right)
$$

where $F^{2}=F F=F_{\mu \nu} F^{\mu \nu}$ and $F^{*} F=F_{\mu \nu}{ }^{*} F^{\mu \nu}$, they read

$$
G_{\mu \nu}=\frac{{ }^{*} F_{\mu \nu}+\frac{1}{4} \lambda\left(F^{*} F\right) F_{\mu \nu}}{\sqrt{1+\frac{1}{2} \lambda F^{2}-\frac{1}{16} \lambda^{2}\left(F^{*} F\right)^{2}}} .
$$

The constitutive relations (2.3) define a nonlinear and higher derivative extension of electromagnetism because we require that setting $\lambda=0$ in (2.3) we recover usual electromagnetism: $G_{\mu \nu}={ }^{*} F_{\mu \nu}$.

We now show that in the general nonlinear case (where the constitutive relations do not involve derivatives of $F$ ) the equations of motion (2.1)-(2.3) can always be obtained from a variational principle provided they satisfy the integrability conditions

$$
\frac{\partial h^{\mu \nu}}{\partial F_{\rho \sigma}}=\frac{\partial h^{\rho \sigma}}{\partial F_{\mu \nu}} .
$$

These conditions are necessary in order to obtain (2.3) from an action $S[F]=\int d^{4} x \mathcal{L}(F)$. Indeed if ${ }^{3} h^{\mu \nu}=2 \frac{\partial \mathcal{L}}{\partial F_{\mu \nu}}$ then (2.7) trivially holds.

In order to show that (2.7) is also sufficient we recall that the field strength $F_{\mu \nu}(x)$ locally is a map from spacetime to $\mathbb{R}^{6}$ (with coordinates $F_{\mu \nu}, \mu<\nu$ ). We assume $h^{\mu \nu}(F, \lambda)$ to be well defined functions on $\mathbb{R}^{6}$ or more in general on an open submanifold $M \subset \mathbb{R}^{6}$

\footnotetext{
${ }^{2}$ Nonlinear and higher derivatives theories of electromagnetism admit one (or more) dimensionful coupling constant(s) $\lambda$. Since the expansions for weak and slowly varying fields are expansions in adimensional variables (like for example $\lambda F F$ and $\lambda F^{*} F$, or, schematically and using a different coupling constant, $\lambda \partial F \partial F)$ we will equivalently say that these expansions are in power series of the coupling constant(s) $\lambda$.

${ }^{3}$ The factor 2 is due to the convention $\frac{\partial F_{\rho \sigma}}{\partial F_{\mu \nu}}=\delta_{\rho}^{\mu} \delta_{\sigma}^{\nu}$ adopted in [7] and in the review [35]. It will be used throughout the paper.
} 
that includes the origin $\left(F_{\mu \nu}=0\right)$ and that is a star shaped region w.r.t. the origin (e.g. a 6 -dimensional ball or cube centered in the origin).

Then condition (2.7) states that the 1-form $\kappa=h^{\mu \nu} d F_{\mu \nu}$ is closed, and hence, by Poincaré lemma, exact on $M$; we write $\hbar=d \mathcal{L}$. We have $\mathcal{L}(F)-\mathcal{L}(0)=\int_{\gamma} \hbar$ for any curve $\gamma(c)$ of coordinates $\gamma_{\mu \nu}(c)$ such that $\gamma_{\mu \nu}(0)=0$ and $\gamma_{\mu \nu}(1)=F_{\mu \nu}$. In particular, choosing the straight line from the origin to the point of coordinates $F_{\mu \nu}$, and setting $S=\int d^{4} x \mathcal{L}(F)$, we immediately obtain

Theorem 1. If the constitutive relations (2.3) do not depend on derivatives of $F$ (i.e. if $\left.h^{\mu \nu}[F, \lambda]=h^{\mu \nu}(F, \lambda)\right)$ and the functions $h^{\mu \nu}(F, \lambda)$ are defined in a star shaped region $M$ (of coordinates $F_{\mu \nu}$ ) and satisfy the integrability conditions $(2.7)$, then the constitutive relations (2.3) are equivalent to the equations ${ }^{3}$

$$
\widetilde{G}^{\mu \nu}=2 \frac{\delta S[F]}{\delta F_{\mu \nu}}
$$

where the action functional $S[F]$ is given by

$$
S[F]=\frac{1}{2} \int d^{4} x F_{\mu \nu} \int_{0}^{1} d c h^{\mu \nu}(c F, \lambda) .
$$

Corollary 2. On spacetimes where closed two forms are exact $(d F=0 \Rightarrow F=d A)$, the equations of motion (2.1)-(2.3) of nonlinear electromagnetism satisfying the conditions of Theorem 1 are equivalent to the equations of motion

$$
\frac{\delta S}{\delta A_{\mu}}=0
$$

where $S=\frac{1}{2} \int d^{4} x \int_{0}^{1} d c F h(c F, \lambda)$.

Proof. Equation (2.1) is the Bianchi identity for $F=d A,(2.3)$ holds because of Theorem 1, and (2.2) is equivalent to the equations of motion (2.10).

We have seen that under the integrability conditions (2.7) locally the equations of motion of nonlinear electromagnetism (2.1)-(2.3) can be obtained from the action

$$
S=\frac{1}{2} \int d^{4} x \int_{0}^{1} d c c F \widetilde{G}_{c}
$$

where $\widetilde{G}_{c}=\frac{1}{c} h(c F, \lambda)$.

It is interesting to generalize these results to the case of nonlinear and higher derivatives electromagnetism. We here present a first step in this direction

Proposition 3. If the equations of motion (2.1)-(2.3) of a nonlinear and higher derivatives electromagnetic theory are obtained from an action functional $S[F]$ then we have

$$
S[F]=\frac{1}{2} \int d^{4} x \int_{0}^{1} d c F h[c F, \lambda]
$$

that we simply rewrite $S=\frac{1}{2} \int d^{4} x \int_{0}^{1} d c c F \widetilde{G}_{c}$. 
Proof. Consider the one parameter family of actions $S_{c}[F]=\frac{1}{c^{2}} S[c F]$. Deriving with respect to $c$ we obtain

$$
-c \frac{\partial S_{c}}{\partial c}=2 S_{c}-\int d^{4} x F \frac{\delta S_{c}[F]}{\delta F}
$$

i.e. $-c \frac{\partial S_{c}}{\partial c}=2 S_{c}-\frac{1}{2} \int d^{4} x F \widetilde{G}_{c}$. It is easy to see that $S_{c}=\frac{1}{2 c^{2}} \int d^{4} x \int_{0}^{c} d c^{\prime} c^{\prime} F \widetilde{G}_{c^{\prime}}$ is the primitive with the correct behaviour under rescaling of $c$ and $F$. We conclude that $\frac{1}{c^{2}} S[c F]=\frac{1}{2 c^{2}} \int d^{4} x \int_{0}^{c} d c^{\prime} c^{\prime} F \widetilde{G}_{c^{\prime}}$, and setting $c=1$ we obtain the thesis.

We now consider the following expansion of an action $S[F]$ even under $F \rightarrow-F$,

$$
S[F]=S^{\{0\}}[F]+S^{\{2\}}[F]+S^{\{4\}}[F]+\ldots
$$

where $S^{\{2 n\}}$ is the term homogeneous in $2 n$ field strengths or their derivatives. Similarly we consider $S_{c}[F]=\frac{1}{c^{2}} S[c F]$ and expand $\widetilde{G}_{c}=2 \frac{\delta S_{c}}{\delta F}$ as

$$
\begin{aligned}
\widetilde{G}_{c} & =\widetilde{G}_{c}^{\{1\}}+\widetilde{G}_{c}^{\{3\}}+\widetilde{G}_{c}^{\{5\}}+\ldots \\
& =\widetilde{G}^{\{1\}}+c^{2} \widetilde{G}^{\{3\}}+c^{4} \widetilde{G}^{\{5\}}+\ldots
\end{aligned}
$$

where $G_{c}^{\{2 n-1\}}$ is the term homogeneous in $2 n-1$ field strengths or their derivatives, and in the second equality we observed that it is also the term proportional to $c^{2 n-2}$ so that $G_{c}^{\{2 n-1\}}=c^{2 n-2} G_{c=1}^{\{2 n-1\}}=c^{2 n-2} G^{\{2 n-1\}}$. Proposition 3 then implies

$$
S^{\{2 n\}}=\frac{1}{4 n} \int d^{4} x F \widetilde{G}^{\{2 n-1\}} .
$$

This expression relates the term in the action proportional to the $2 n^{\text {th }}$ power of $F$ or its derivatives, to the term in $\widetilde{G}$ proportional to the $(2 n-1)^{\text {th }}$ power of $F$ or its derivatives.

Note 4. Expression $S=\frac{1}{2} \int d^{4} x \int_{0}^{1} d c c F \widetilde{G}_{c}$, in the equivalent form

$$
S=\frac{1}{4} \int d^{4} x \int_{0}^{1} d \kappa F \widetilde{G}_{\kappa}
$$

(where $\kappa=c^{2}$ ) has been considered for self-dual theories in [27] and called reconstruction identity. It has been used, together with an expression equivalent to (2.16), to reconstruct the action $S$ from equations of motion with duality rotation symmetry in examples with higher derivatives of $F$.

Note 5. In appendix $\mathrm{C}$ we show that for nonlinear theories without higher derivatives, the 1.h.s. and r.h.s. of (2.13) are half the spacetime integral of the trace of the energy momentum tensor.

\section{$2.2 \quad \mathrm{U}(1)$ duality rotations}

Nonlinear and higher derivatives electromagnetism admits $U(1)$ duality rotation symmetry if given a field configuration $F, G$ that satisfies (2.1)-(2.3) then the rotated configuration

$$
\left(\begin{array}{l}
F^{\prime} \\
G^{\prime}
\end{array}\right)=\left(\begin{array}{cc}
\cos \alpha & -\sin \alpha \\
\sin \alpha & \cos \alpha
\end{array}\right)\left(\begin{array}{l}
F \\
G
\end{array}\right),
$$


that is trivially a solution of $\partial_{\mu} \widetilde{F}^{\mu \nu}=0, \partial_{\mu} \widetilde{G}^{\mu \nu}=0$, satisfies also $\widetilde{G}_{\mu \nu}^{\prime}=h_{\mu \nu}\left[F^{\prime}, \lambda\right]$, so that $F^{\prime}, G^{\prime}$ is again a solution of the equations of motion. If we consider an infinitesimal duality rotation, $F \rightarrow F+\Delta F, G \rightarrow G+\Delta G$ then condition $\widetilde{G}_{\mu \nu}^{\prime}=h_{\mu \nu}\left[F^{\prime}, \lambda\right]$ reads $\Delta \widetilde{G}_{\mu \nu}=\int d^{4} x \frac{\delta h_{\mu \nu}}{\delta F_{\rho \sigma}} \Delta F^{\rho \sigma}$, i.e., $\widetilde{F}_{\mu \nu}=-\int d^{4} x \frac{\delta h_{\mu \nu}}{\delta F_{\rho \sigma}} G^{\rho \sigma}$, that we simply rewrite

$$
\widetilde{F}_{\mu \nu}=-\int d^{4} x \frac{\delta \widetilde{G}_{\mu \nu}}{\delta F_{\rho \sigma}} G^{\rho \sigma}
$$

It is straightforward to check that electromagnetism and Born-Infeld theory satisfy (2.19).

If the theory is obtained from an action functional $S[F]$ (in the field strength $F$ and its derivatives) then (2.3) is given by

$$
\widetilde{G}^{\mu \nu}=2 \frac{\delta S[F]}{\delta F_{\mu \nu}} .
$$

In particular it follows that

$$
\frac{\delta \widetilde{G}^{\mu \nu}}{\delta F_{\rho \sigma}}=\frac{\delta \widetilde{G}^{\rho \sigma}}{\delta F_{\mu \nu}},
$$

hence the duality symmetry condition (or self-duality condition) (2.19) equivalently reads $\widetilde{F}_{\mu \nu}=-\int d^{4} x \frac{\delta \widetilde{G}_{\rho \sigma}}{\delta F_{\mu \nu}} G^{\rho \sigma}$. Now writing $\widetilde{F}_{\mu \nu}=\frac{\delta}{\delta F_{\mu \nu}} \frac{1}{2} \int d^{4} x F_{\rho \sigma} \widetilde{F}^{\rho \sigma}$ we equivalently have

$$
\frac{\delta}{\delta F_{\mu \nu}} \int d^{4} x F \widetilde{F}+G \widetilde{G}=0
$$

where $F \widetilde{F}=F_{\rho \sigma} \widetilde{F}^{\rho \sigma}$ and similarly for $G \widetilde{G}$. We require this condition to hold for any field configuration $F$ (i.e. off shell of (2.1), (2.2)) and hence we obtain the Noether-GaillardZumino (NGZ) self-duality condition ${ }^{4}$

$$
\int d^{4} x F \widetilde{F}+G \widetilde{G}=0 .
$$

The vanishing of the integration constant is determined for example by the condition $G={ }^{*} F$ for weak and slowly varying fields, i.e. by the condition that in this regime the theory is approximated by usual electromagnetism.

We also observe that the NGZ self-duality condition (2.23) is equivalent to the invariance of $S^{\text {inv }}=S-\frac{1}{4} \int d^{4} x F \widetilde{G}$, indeed under a rotation (2.18) with infinitesimal parameter $\alpha$ we have $S^{\operatorname{inv}}\left[F^{\prime}\right]-S^{\text {inv }}[F]=-\frac{\alpha}{4} \int d^{4} x F \widetilde{F}+G \widetilde{G}=0$.

Note 6. If the Lagrangian $L(F)$ of the action functional $S[F]$ does not depend on the derivatives of $F$, then we cannot integrate by parts and condition (2.23) is equivalent to

$$
F \widetilde{F}+G \widetilde{G}=0
$$

\footnotetext{
${ }^{4}$ Note that (2.23) (the integrated form of (2.24)) also follows in a straightforward manner by repeating the passages in [7] but with $G$ the functional derivative of the action rather than the partial derivative of the lagrangian $[13,35]$. This makes a difference for nonlinear theories which also contain terms in derivatives of $F$.
} 
since the field configuration $F$ is arbitrary (and therefore with arbitrary support in spacetime). On shell of (2.1), (2.2) we can introduce the electric potential $A_{\mu}$ and the magnetic one $B_{\mu}$ so that $F_{\mu \nu}=\partial_{\mu} A_{\nu}-\partial_{\nu} A_{\mu}, G_{\mu \nu}=\partial_{\mu} B_{\nu}-\partial_{\nu} B_{\mu}$ and (2.24) becomes the (NoetherGaillard-Zumino) current conservation condition $\partial_{\mu} J^{\mu}=\partial_{\mu}\left(A_{\nu} \widetilde{F}^{\mu \nu}+B_{\nu} \widetilde{G}^{\mu \nu}\right)=0$.

Examples of theories satisfying (2.23) and not (2.24) are obtained in appendix A, where we generalize the example presented in [25].

Note 7. If the Lagrangian $L(F)$ is in Minkowski spacetime and if it depends only on $F$ and not on its derivatives, then Lorentz invariance implies that it depends on the scalars $F F$ and $(F \widetilde{F})^{2}$, where the square in $(F \widetilde{F})^{2}$ is needed for parity symmetry (space inversion invariance). More in general we can consider a Lagrangian in curved spacetime that depends only on the (pseudo)scalars $F F$ and $F^{*} F$. It is then possible to integrate the differential equation (2.24): $F_{\mu \nu}{ }^{*} F^{\mu \nu}-4^{*}\left(\frac{\partial L}{\partial F^{\mu \nu}}\right) \frac{\partial L}{\partial F_{\mu \nu}}=0$. The solution is presented in [34] (and an alternative form is presented in [36], see also [32]), it depends on an arbitrary real valued function $v(s)$ of a real variable $s$, with the initial condition that in the limit $s \rightarrow 0$ then $v(s) \rightarrow-s$. However $L(F)$ is explicitly determined only after inverting a function related to $v(s)$. Hence explicit solutions $L(F)$ in terms of simple functions are very difficult to be found.

This suggests to look for solutions $L(F)$, and more in general actions $S[F]$, that are power series in the coupling constant $\lambda$.

Note 8. Given an action $S[F]$ with self-dual equations of motion the one parameter family of theories defined by $S_{c}[F]=\frac{1}{c^{2}} S[c F]$ (with $c \geq 0$, cf. end of section 2.1) are also self-dual. This is so because for any given value of $c$ the action $S_{c}[F]$ satisfies the corresponding NGZ self-duality conditions (2.23):

$$
\int d^{4} x F \widetilde{F}-2 \frac{\delta S_{c}[F]}{\delta F} 2 \frac{\widetilde{\delta S_{c}[F]}}{\delta F}=0
$$

Indeed $\frac{\delta S_{c}[F]}{\delta F} \frac{\widetilde{\delta S_{c}[F]}}{\delta F}=\frac{1}{c^{4}} \frac{\delta S[c F]}{\delta F} \widehat{\frac{\delta S[c F]}{\delta F}}=\frac{1}{c^{2}} \frac{\delta S[c F]}{\delta c F} \frac{\widetilde{\delta S[c F]}}{\delta c F}$. Therefore condition (2.25) is equivalent to $\int d^{4} x c F c \widetilde{F}-2 \frac{\delta S[c F]}{\delta c F} 2 \frac{\widehat{\delta S[c F]}}{\delta c F}=0$. These are the self-duality conditions for the action $S[\hat{F}]$ with $\hat{F}=c F$. Hence these conditions hold because the self-duality conditions for the initial action $S$ hold for any field configuration.

This result allows to provide jet another derivation of the invariance under duality rotation of expression (2.13) for self-dual actions: One has just to recall that the variation of the action with respect to a duality invariant parameter is duality invariant [7].

\subsection{Complex and chiral variables}

Following Schrödinger $[1,34]$ it is convenient to consider the complex variables

$$
T=F-i G, \quad \bar{T}=F+i G,
$$

that under duality transform with a phase: $T \rightarrow e^{-i \alpha} T, \bar{T} \rightarrow e^{i \alpha} \bar{T}$, and that treat on an equal footing the electric and magnetic field strengths $F$ and $G$. In the new variables the 
NGZ self-duality condition (2.23) reads $\int d^{4} x \bar{T} \widetilde{T}=0$, or equivalently

$$
\int d^{4} x \sqrt{g} \bar{T}^{*} T=0 .
$$

Following [25] we further consider the complex (anti)selfdual combinations $F^{ \pm}=\frac{1}{2}(F \pm$ $\left.i^{*} F\right), G^{ \pm}=\frac{1}{2}\left(G \pm i^{*} G\right)$ and

$$
\begin{aligned}
T^{+} & =\frac{1}{2}\left(T+i^{*} T\right)=F^{+}-i G^{+}, & T^{-} & =\frac{1}{2}\left(T-i^{*} T\right)=F^{-}-i G^{-}, \\
\overline{T^{+}} & =\frac{1}{2}\left(\bar{T}-i^{*} \bar{T}\right)=F^{-}+i G^{-}=\bar{T}^{-}, & \overline{T^{-}} & =\frac{1}{2}\left(\bar{T}+i^{*} \bar{T}\right)=F^{+}+i G^{+}=\bar{T}^{+} .
\end{aligned}
$$

The fields in the first row have duality charge +1 because transform with $e^{-i \alpha}$ under the duality rotation (2.18), while their complex conjugates in the second row have duality charge -1 . Complex conjugation inverts chirality hence $T^{+}$and $\bar{T}^{-}=\bar{T}^{+}$have chirality +1 while $T^{-}$and $\overline{T^{+}}=\bar{T}^{-}$have chirality -1 .

The (anti)selfdual combinations have definite behaviour in the coupling constant $\lambda \rightarrow 0$ limit. Since in this limit we recover usual electromagnetism we have $G \rightarrow{ }^{*} F$ and $G^{ \pm} \rightarrow$ $\mp i F^{ \pm}$, and hence

$$
T^{+} \rightarrow 0, T^{-} \rightarrow 2 F^{-} .
$$

The NGZ self-duality condition $(2.23)$ in these variables reads $\left(\right.$ use $\left.\left({ }^{*} T\right)^{ \pm}={ }^{*}\left(T^{ \pm}\right)=\mp i T^{ \pm}\right)$

$$
\int d^{4} x \sqrt{g} T^{+} \overline{T^{-}}-\overline{T^{+}} T^{-}=0 .
$$

\subsection{The action functional $\mathcal{I}\left[T^{-}, \overline{T^{-}}\right]$}

As noticed in [26], the Bossard and Nicolai proposal [25] for constructing self-dual equations of motions is easily expressed in terms of chiral variables: We consider a real valued functional $\mathcal{I}\left[T^{-}, \overline{T^{-}}\right]$in the chiral variables ${ }^{5} T^{-}, \overline{T^{-}}$and define the constitutive relations (called deformed twisted self duality conditions in [25], and nonlinear twisted self-duality conditions in $[26])$

$$
T^{+\mu \nu}=\frac{1}{\sqrt{g}} \frac{\delta \mathcal{I}\left[T^{-}, \overline{T^{-}}\right]}{\delta \overline{T^{-}}{ }_{\mu \nu}}, \quad \overline{T^{+}} \mu \nu=\frac{1}{\sqrt{g}} \frac{\delta \mathcal{I}\left[T^{-}, \overline{T^{-}}\right]}{\delta T_{\mu \nu}^{-}} .
$$

Reality $^{6}$ of $\mathcal{I}$ implies that the second equation is just the complex conjugate of the first one, hence the constitutive relations are 6 real equations as in (2.3) and in (2.20). If moreover $\mathcal{I}$ is duality invariant under $T^{-} \rightarrow e^{-i \alpha} T^{-}, \overline{T^{-}} \rightarrow e^{i \alpha} \overline{T^{-}}$then relations (2.32)

\footnotetext{
${ }^{5}$ We stress that the independent variables in $\mathcal{I}$ are $T^{-}$and its complex conjugate $\overline{T^{-}}$, just like in $S[F]$ or $S\left[F^{-}, F^{+}\right]$the independent variables are $F^{-}$and its complex conjugate $F^{+}$. The variables $T^{+}, \overline{T^{+}}$(and hence $T, \bar{T})$ are then defined in terms of the $T^{-}, \overline{T^{-}}$ones.

${ }^{6}$ The reality condition is $\mathcal{I}\left[T^{-}, \overline{T^{-}}\right]=\overline{\mathcal{I}\left[T^{-}, \overline{T^{-}}\right]}$. Then we extend $\mathcal{I}\left[T^{-}, \overline{T^{-}}\right]$to $\widehat{\mathcal{I}}\left[T^{-}, \overline{U^{-}}\right] \equiv$ $\frac{1}{2}\left(\mathcal{I}\left[T^{-}, \overline{U^{-}}\right]+\overline{\mathcal{I}\left[U^{-}, \overline{T^{-}}\right]}\right)$that by construction satisfies $\overline{\widehat{\mathcal{I}}\left[T^{-}, \overline{U^{-}}\right]}=\widehat{\mathcal{I}}\left[U^{-}, \overline{T^{-}}\right]$for arbitrary complex and independent fields $T^{-}$and $U^{-}$. The functional variation in (2.32), where $\overline{T^{-}}$is kept independent from $T^{-}$, then explicitly reads $T^{+}=\left.\frac{1}{\sqrt{g}} \frac{\delta \widehat{\mathcal{I}}\left[T^{-}, \overline{U^{-}}\right]}{\delta \overline{U^{-}}}\right|_{U^{-}=T^{-}}, \overline{T^{+}}=\left.\frac{1}{\sqrt{g}} \frac{\delta \widehat{\mathcal{I}}\left[T^{-}, \overline{U^{-}}\right]}{\delta T^{-}}\right|_{U^{-}=T^{-}}$.
} 
imply the NGZ self-duality condition (2.23); indeed under an infinitesimal duality rotation $T^{-} \rightarrow T^{-}+\Delta T^{-}, \Delta T^{-}=-i \alpha T^{-}$we have:

$$
0=\Delta \mathcal{I}=\int d^{4} x \frac{\delta \mathcal{I}}{\delta \overline{T^{-}}} \Delta \overline{T^{-}}+\frac{\delta \mathcal{I}}{\delta T^{-}} \Delta T^{-}=i \alpha \int d^{4} x \sqrt{g} T^{+} \overline{T^{-}}-\overline{T^{+}} T^{-} .
$$

This is a powerful approach because the constitutive relations are easily given (though the dependence $\widetilde{G}_{\mu \nu}=h_{\mu \nu}[F, \lambda]$ is determined implicitly), and the self-duality condition is also easily implemented: just consider duality invariant functionals $\mathcal{I}$. Furthermore, Lorentz (or, in curved spacetime, diffeomorphisms) invariance of the functional $\mathcal{I}$ implies Lorentz (diffeomorphisms) covariance of the nonlinear and higher derivatives equations of motion.

The problem with this approach is that of finding an action functional $S[F]$ such that the constitutive relations $(2.20):{ }^{*} G^{\mu \nu}=\frac{2}{\sqrt{g}} \frac{\delta S[F]}{\delta F_{\mu \nu}}$, are equivalent to the constitutive relations (2.32).

We first approach this problem perturbatively, and give explicit expressions for the lowest order perturbations; in the next section we solve the problem (albeit implicitly) by using a Legendre transform between $S$ and $\mathcal{I}$.

In the perturbative approach we assume that $\mathcal{I}=\mathcal{I}\left[T^{-}, \overline{T^{-}}\right]$is a power series in the coupling constant $\lambda$,

$$
\mathcal{I}\left[T^{-}, \overline{T^{-}}\right]=\mathcal{I}^{[0]}\left[T^{-}, \overline{T^{-}}\right]+\mathcal{I}^{[1]}\left[T^{-}, \overline{T^{-}}\right]+\mathcal{I}^{[2]}\left[T^{-}, \overline{T^{-}}\right]+\ldots
$$

where $\mathcal{I}^{[n]}$ denotes the term proportional to $\lambda^{n}$, and in this expansion $T^{-}, \overline{T^{-}}$are considered the elementary independent fields (and hence $\lambda$ independent).

Then $S[F]=S\left[F^{-}, F^{+}\right]$is found as a power series

$$
S\left[F^{-}, F^{+}\right]=S^{(0)}\left[F^{-}, F^{+}\right]+S^{(1)}\left[F^{-}, F^{+}\right]+S^{(2)}\left[F^{-}, F^{+}\right]+\ldots
$$

where $S^{(n)}$ denotes the term proportional to $\lambda^{n}$, and in this expansion $F^{-}, F^{+}$are the elementary independent fields (and hence $\lambda$ independent). The initial condition is $\mathcal{I}^{[0]}=0$, that corresponds to linear electromagnetism, $S^{(0)}=-\frac{1}{4} \int d^{4} x \sqrt{g} F^{2}$.

Since $\overline{T^{+}}=F^{-}+i G^{-}$implies ${\overline{T^{+}}}^{(n)}=i G^{-(n)}$ for $n \geq 1$, we see that equivalence of the constitutive relations (2.32) and (2.20), that we rewrite as $G^{ \pm \mu \nu}=\frac{ \pm 2 i}{\sqrt{g}} \frac{\delta S}{\delta F_{\mu \nu}^{ \pm}}$, is obtained by requiring order by order in $n$ that the term $S^{(n)}$ satisfies the condition

$$
2 \frac{\delta S^{(n)}}{\delta F_{\mu \nu}^{-}}=\left(\left.\frac{\delta \mathcal{I}}{\delta T_{\mu \nu}^{-}}\right|_{\frac{T^{-}\left[F^{-}, F^{+}\right]}{T^{-}\left[F^{-}, F^{+}\right]}}\right)^{(n)}
$$

where on the right hand side we consider $\frac{\delta \mathcal{I}}{\delta T^{-}}$as a functional of $F^{-}$and $F^{+}$because $T^{-}=T^{-}\left[F^{-}, F^{+}\right]$; the dependence $T^{-}=T^{-}\left[F^{-}, F^{+}\right]$being implicitly determined by the chiral variables constitutive relations $(2.32)$ and the relations $T^{ \pm}=F^{ \pm}-i G^{ \pm}$, that, in order to stress that the independent variables are $T^{-}$and $\overline{T^{-}}$, we rewrite as

$$
\begin{aligned}
2 F^{-} & =T^{-}+\frac{1}{\sqrt{g}} \frac{\delta \mathcal{I}\left[T^{-}, \overline{T^{-}}\right]}{\delta T_{\mu \nu}^{-}}, & 2 F^{+} & =\frac{1}{\sqrt{g}} \frac{\delta \mathcal{I}\left[T^{-}, \overline{T^{-}}\right]}{\delta \overline{T^{-}} \mu \nu}+\overline{T^{-}}, \\
-2 i G^{-} & =T^{-}-\frac{1}{\sqrt{g}} \frac{\delta \mathcal{I}\left[T^{-}, \overline{T^{-}}\right]}{\delta T_{\mu \nu}^{-}}, & -2 i G^{+} & =\frac{1}{\sqrt{g}} \frac{\delta \mathcal{I}\left[T^{-}, \overline{T^{-}}\right]}{\delta \overline{T^{-}}{ }_{\mu \nu}}-\overline{T^{-}} .
\end{aligned}
$$


In appendix $\mathrm{B}$ we determine the first two nontrivial terms of the nonlinear and higher derivatives electromagnetic action associated with an arbitrary functional $\mathcal{I}=\mathcal{I}^{[0]}+\mathcal{I}^{[1]}+$ $\mathcal{I}^{[2]}+\ldots$, with $\mathcal{I}^{[0]}=0$. They read

$$
\begin{aligned}
& S^{(1)}\left[F^{-}, F^{+}\right]=\frac{1}{4} \mathcal{I}^{[1]}\left[2 F^{-}, 2 F^{+}\right], \\
& S^{(2)}\left[F^{-}, F^{+}\right]=\frac{1}{4} \mathcal{I}^{[2]}\left[2 F^{-}, 2 F^{+}\right]-\frac{1}{2} \int d^{4} x \frac{1}{\sqrt{g}} \frac{\delta S^{(1)}}{\delta F^{-}} \frac{\delta S^{(1)}}{\delta F^{-}}+\frac{\delta S^{(1)}}{\delta F^{+}} \frac{\delta S^{(1)}}{\delta F^{+}} .
\end{aligned}
$$

We recall that at zeroth order $S^{(0)}\left[F^{-}, F^{+}\right]=-\frac{1}{4} \int d^{4} x \sqrt{g} F^{-2}+F^{+2}=-\frac{1}{4} \int d^{4} x \sqrt{g} F^{2}$.

\subsection{From $S[F]$ to $\mathcal{I}\left[T^{-}, \overline{T^{-}}\right]$via Legendre transform}

We now show, as in [32], that $\mathcal{I}\left[T^{-}, \overline{T^{-}}\right]$and $S[F]$ are related by

$$
\frac{1}{4} \mathcal{I}\left[T^{-}, \overline{T^{-}}\right]=S[F]+\int d^{4} x \sqrt{g} \frac{1}{2} T^{-} F^{-}-\frac{1}{8} T^{-2}-\frac{1}{4} F^{-2}+\frac{1}{2} \overline{T^{-}} F^{+}-\frac{1}{8}{\overline{T^{-}}}^{2}-\frac{1}{4} F^{+2} .
$$

This is actually a Legendre transform, and it implies that the constitutive relations (2.32) are equivalent to the constitutive relations (2.20), i.e., $G^{ \pm \mu \nu}=\frac{ \pm 2 i}{\sqrt{g}} \frac{\delta S\left[F^{-}, F^{+}\right]}{\delta F_{\mu \nu}^{ \pm}}$.

In order to recognize (2.40) as a Legendre transform we define the functional

$$
U\left[F^{-}, F^{+}\right]=-2 S\left[F^{-}, F^{+}\right]+\frac{1}{2} \int d^{4} x \sqrt{g} F^{-2}+F^{+2} .
$$

Recalling that $i G^{-}=F^{-}-T^{-}($see $(2.28))$ the constitutive relations $G^{ \pm \mu \nu}=\frac{ \pm 2 i}{\sqrt{g}} \frac{\delta S\left[F^{-}, F^{+}\right]}{\delta F_{\mu \nu}^{ \pm}}$ now read

$$
T^{-}=\frac{1}{\sqrt{g}} \frac{\delta U}{\delta F^{-}}, \quad \overline{T^{-}}=\frac{1}{\sqrt{g}} \frac{\delta U}{\delta F^{+}} .
$$

These relations (at least for weak and slowly varying fields) implicitly determine $F^{ \pm}=$ $F^{ \pm}\left[T^{-}, \overline{T^{-}}\right]$. We then consider the Legendre transform

$$
V\left[T^{-}, \overline{T^{-}}\right]=-U\left[F^{-}, F^{+}\right]+\int d^{4} x \sqrt{g} T^{-} F^{-}+\overline{T^{-}} F^{+} .
$$

Varying w.r.t. $T^{-}$and $\overline{T^{-}}$we obtain that the dependence $F^{ \pm}=F^{ \pm}\left[T^{-}, \overline{T^{-}}\right]$is given by

$$
F^{-}=\frac{1}{\sqrt{g}} \frac{\delta V}{\delta T^{-}}, \quad F^{+}=\frac{1}{\sqrt{g}} \frac{\delta V}{\delta \overline{T^{-}}} .
$$

Therefore (2.44) are the inverse relations of (2.42), in particular they are equivalent to $G^{ \pm \mu \nu}=\frac{ \pm 2 i}{\sqrt{g}} \frac{\delta S\left[F^{-}, F^{+}\right]}{\delta F_{\mu \nu}^{ \pm}}$. We now define the functional $\mathcal{I}\left[T^{-}, \overline{T^{-}}\right]$via

$$
V\left[T^{-}, \overline{T^{-}}\right]=\frac{1}{2} \mathcal{I}\left[T^{-}, \overline{T^{-}}\right]+\frac{1}{4} \int d^{4} x \sqrt{g} T^{-2}+{\overline{T^{-}}}^{2} .
$$

Relation (2.40) is trivially equivalent to (2.43). Furthermore the constitutive relations $G^{ \pm \mu \nu}=\frac{ \pm 2 i}{\sqrt{g}} \frac{\delta S\left[F^{-}, F^{+}\right]}{\delta F_{\mu \nu}^{ \pm}}$and (2.32) are equivalent because (2.44) is easily seen to be equivalent to $(2.37)$, i.e., to $(2.32)$. 
Let's now study duality rotations. We consider $F$ to be the elementary fields and let $S[F]$ give self-dual constitutive relations. Under infinitesimal duality rotations (2.18), $F \rightarrow F+\Delta F=F-\alpha G, G \rightarrow G+\Delta G=G+\alpha F$ we have (since $T^{-}=F^{-}-\frac{2}{\sqrt{g}} \frac{\delta S}{\delta F^{-}}$) that $T^{-} \rightarrow T^{-}+\Delta T^{-}=T^{-}-i \alpha T^{-}$. We calculate the variation of (2.40) under duality rotations. After a little algebra we see that

$$
\begin{aligned}
\Delta \mathcal{I} & =\mathcal{I}\left[T^{-}+\Delta T^{-}, \overline{T^{-}}+\Delta \overline{T^{-}}\right]-\mathcal{I}\left[T^{-}, \overline{T^{-}}\right] \\
& =S[F+\Delta F]-S[F]+\frac{\alpha}{4} \int d^{4} x \sqrt{g} G \widetilde{G}-F \widetilde{F}=-\frac{\alpha}{4} \int d^{4} x \sqrt{g} G \widetilde{G}+F \widetilde{F}=0
\end{aligned}
$$

where we used that $S[F+\Delta F]-S[F]=\int d^{4} x \frac{\delta S}{\delta F} \Delta F=-\frac{\alpha}{2} \int d^{4} x \widetilde{G} G$, and the self-duality conditions (2.23). Hence $\mathcal{I}$ is invariant under duality rotations.

Vice versa, we can consider $T^{-}, \overline{T^{-}}$to be the elementary fields and assume $\mathcal{I}\left[T^{-}, \overline{T^{-}}\right]$ to be duality invariant. Then from (2.37) and $i G^{-}=F^{-}-T^{-}$, i.e., form (2.37) and (2.38), it follows that under the infinitesimal rotation $T^{-} \rightarrow T^{-}+\Delta T^{-}=T^{-}-i \alpha T^{-}$we have $F \rightarrow F+\Delta F=F-\alpha G, G \rightarrow G+\Delta G=G+\alpha F$, and from (2.46) we recover the self-duality conditions (2.23) for the action $S[F]$.

This shows the equivalence betweeen the $S[F]$ and the $\mathcal{I}\left[T^{-}, \overline{T^{-}}\right]$formulations of selfdual constitutive relations. Hence the deformed twisted self-duality condition proposal originated in the context of supergravity counterterms is actually the general framework needed to discuss self-dual theories starting from a variational principle.

\section{Constitutive relations without self-duality}

The constitutive relations (2.3) or (2.20) express $G$ as a function of $F$ and its derivatives. They do not treat on equal footing $F$ and $G$ and therefore their eventual compatibility with duality symmetry is hidden. On the other hand the independent chiral variables $T^{-}, \overline{T^{-}}$ of the constitutive relations (2.32) (the deformed twisted self duality conditions) treat by construction $F$ and $G$ on equal footing, and duality rotations are simply implemented via multiplication by a phase. There however the relation betweeen $G$ and $F$ is implicitly given. Moreover, already the description of Born-Infeld theory is quite nontrivial in these chiral variables. We here further study the nonlinear relations between these two formulations and related ones. This study sheds light on the structure of self-dual theories, in particular it will lead to a closed form expression of the constitutive relations (2.32) for the BornInfeld theory.

We proceed with a manifestly duality symmetric reformulation of the constitutive relations (2.3) (and more precisely of the relations (3.1) below). This is achieved doubling them (to ${ }^{*} G=h[F, \lambda]$ and ${ }^{*} F=k[G, \lambda]$ ) and then constraining them via a symplectic matrix $\mathcal{M}$. This matrix is well known in the study of duality rotations in linear electromagnetism coupled to scalar fields (see e.g. [35]). Here $\mathcal{M}$ will be in general dependent on the field strengths $F, G$ and their derivatives, leading to nonlinear and higher derivatives electromagnetism. Its structure will be fully determined only by requiring that the doubled constitutive relations consistently give just 6 independent equations that determine $G$ in 
terms of $F$ and vice versa. Notice that even thought our aim is the study of self-dual theories, in this section we do not assume that the constitutive relations are compatible with duality symmetry.

The constraints on the $\mathcal{M}$ matrix are then analized in terms of the Schrödinger's variables $T, \bar{T}$. It is in these variables that Born-Infeld theory has an extemely simple description $[1,34]$.

\subsection{The $\mathcal{N}$ and $\mathcal{M}$ matrices}

More insights in the constitutive relations (2.3) can be obtained if we restrict our study to the wide subclass that can be written as

$$
{ }^{*} G_{\mu \nu}=\mathcal{N}_{2} F_{\mu \nu}+\mathcal{N}_{1}{ }^{*} F_{\mu \nu}
$$

where $\mathcal{N}_{2}$ is a real scalar field, while $\mathcal{N}_{1}$ is a real pseudo-scalar field (i.e., it is not invariant under parity, or, if we are in curved spacetime, it is not invariant under an orientation reversing coordinate transformation). Explicit examples of more general constitutive relations are in appendix A. As usual in the literature we set

$$
\mathcal{N}=\mathcal{N}_{1}+i \mathcal{N}_{2}
$$

then, relations (3.1) are equivalent to $G^{+}=\mathcal{N} F^{+}$. In nonlinear theories $\mathcal{N}$ depends on the field strength $F$, and in higher derivative theories also on derivatives of $F$, we have therefore in general a functional dependence $\mathcal{N}=\mathcal{N}[F, \lambda]$. Furthermore $\mathcal{N}$ is required to satisfy $\mathcal{N} \rightarrow-i$ in the limit $\lambda \rightarrow 0$ so that we recover classical electromagnetism when the coupling constant $\lambda \rightarrow 0$, or otherwise stated, in the weak and slowly varying field limit, i.e., when we discard higher powers of $F$ and derivatives of $F$. We also assume that $\mathcal{N}$ can be expanded in power series of the coupling constant ${ }^{7} \lambda$ (we will relax this assumption in Note 11). Then, since $\mathcal{N}_{2}=-1+O(\lambda), \mathcal{N}_{2}$ is invertible, and from relation (3.1) we obtain $\widetilde{F}=\mathcal{N}_{2}^{-1} \mathcal{N}_{1} F-\mathcal{N}_{2}^{-1} G$ and $\widetilde{G}=\mathcal{N}_{2} F+\mathcal{N}_{1} \mathcal{N}_{2}^{-1} \mathcal{N}_{1} F-\mathcal{N}_{1} \mathcal{N}_{2}^{-1} G$ so that the constitutive relation (3.1) is equivalent to the more duality symmetric one

$$
\left(\begin{array}{l}
{ }^{*} F \\
{ }^{*} G
\end{array}\right)=\left(\begin{array}{cc}
0 & -1 \\
1 & 0
\end{array}\right) \mathcal{M}\left(\begin{array}{l}
F \\
G
\end{array}\right)
$$

where the matrix $\mathcal{M}$ is given by

$$
\mathcal{M}(\mathcal{N})=\left(\begin{array}{cc}
1 & -\mathcal{N}_{1} \\
0 & 1
\end{array}\right)\left(\begin{array}{cc}
\mathcal{N}_{2} & 0 \\
0 & \mathcal{N}_{2}^{-1}
\end{array}\right)\left(\begin{array}{cc}
1 & 0 \\
-\mathcal{N}_{1} & 1
\end{array}\right)=\left(\begin{array}{cc}
\mathcal{N}_{2}+\mathcal{N}_{1} \mathcal{N}_{2}^{-1} \mathcal{N}_{1} & -\mathcal{N}_{1} \mathcal{N}_{2}^{-1} \\
-\mathcal{N}_{2}^{-1} \mathcal{N}_{1} & \mathcal{N}_{2}^{-1}
\end{array}\right)
$$

Finally, in order to really treat on equal footing the electric and magnetic field strengths $F$ and $G$, we should consider functionals $N_{1}[F, G, \lambda]$ and $N_{2}[F, G, \lambda]$ such that the constitutive

\footnotetext{
${ }^{7} \mathrm{By} \lambda$ we can denote also more than one coupling constant. For example when a nonlinear theory in flat space is generalized to a curved background there naturally appears a new coupling related to the background curvature. Similarly, as already said, if the theory has higher derivatives so that it can be expanded in appropriate powers of derivatives of $F$.
} 
relations ${ }^{*} G=N_{2}[F, G, \lambda] F+N_{1}[F, G, \lambda]^{*} F$ are equivalent to (3.1), i.e., such that on shell of these relations, $N_{1}[F, G, \lambda]=\mathcal{N}_{1}[F, \lambda]$ and $N_{2}[F, G, \lambda]=\mathcal{N}_{2}[F, \lambda]$.

Since we assume $N_{1}[F, G, \lambda]$ and $N_{2}[F, G, \lambda]$ to be power series in $\lambda$ with $N_{1}=O(\lambda)$ and $N_{2}=-1+O(\lambda)$ the constitutive relations ${ }^{*} G=N_{2}[F, G, \lambda] F+N_{1}[F, G, \lambda]{ }^{*} F$ are well given in the sense that they are always equivalent to the ${ }^{*} G=\mathcal{N}_{2}[F, \lambda] F+\mathcal{N}_{1}[F, \lambda]^{*} F$ ones (just expand in power series of $\lambda$ and iteratively substitute $G$ in $N_{1}[F, G, \lambda]$ and $N_{2}[F, G, \lambda]$ ).

Henceforth, with slight abuse of notation, from now on the $\mathcal{N}, \mathcal{N}_{1}, \mathcal{N}_{2}$ fields in (3.1)(3.4) will in general be functionals of both $F$ and $G$.

The matrix $\mathcal{M}(\mathcal{N})$ in (3.4) is symmetric and symplectic (it has indeed determinant equal to 1$)$. The space of symmetric and symplectic matrices has two disconnected components, that of positive definite and of negative definite matrices. $\mathcal{M}(\mathcal{N})$ is negative definite because $\mathcal{N}_{2}^{-1} \rightarrow-1+O(\lambda)$. Recalling that any symmetric, symplectic and negative definite $2 \times 2$ matrix is of the kind (3.4) with $\mathcal{N}_{1}$ real and $\mathcal{N}_{2}$ real and negative (for a proof see for example the review [35], appendix $\mathrm{A}$, where it is also shown that $\mathcal{M}$ and $\mathcal{N}=\mathcal{N}_{1}+i \mathcal{N}_{2}$ parametrize the coset space $\operatorname{Sp}(2, \mathbb{R}) / \mathrm{U}(1))$, we have that

Proposition 9. Any symmetric and symplectic $2 \times 2$ matrix $\mathcal{M}$ that has a power series expansion in $\lambda$ with $\mathcal{M}=-1+O(\lambda)$ is of the kind (3.4) with $\mathcal{N}_{1}=O(\lambda)$ real and $\mathcal{N}_{2}=-1+O(\lambda)$ real.

We now reverse the argument that led from (3.1) to (3.3). We consider constitutive relations of the form

$$
\left(\begin{array}{l}
{ }^{*} F \\
{ }^{*} G
\end{array}\right)=\left(\begin{array}{cc}
0 & -1 \\
1 & 0
\end{array}\right) \mathcal{M}[F, G, \lambda]\left(\begin{array}{l}
F \\
G
\end{array}\right)
$$

that treat on equal footing $F$ and $G$, and where $\mathcal{M}=\mathcal{M}[F, G, \lambda]$ is now an arbitrary real $2 \times 2$ matrix (with scalar entries $\mathcal{M}_{i j}$ ). We require $\mathcal{M}=-1+O(\lambda)$ so that we recover classical electromagnetism when the coupling constant $\lambda \rightarrow 0$. A priory (3.5) is a set of 12 real equations, twice as much as those present in the constitutive relations (3.1). We want only 6 of these 12 relations to be independent so to be able to determine $G$ in terms of independent fields $F$ (or equivalently $F$ in terms of independent fields $G$ ). Only in this case the constitutive relations are well given.

Proposition 10. The constitutive relations (3.5) with $\mathcal{M}[F, G, \lambda]=-1+O(\lambda)$ are well given if and only if on shell of (3.5) the matrix $\mathcal{M}[F, G, \lambda]$ is symmetric and symplectic.

Proof. i) Let $\mathcal{M}[F, G, \lambda]=-1+O(\lambda)$ be symmetric and symplectic on shell of (3.5). Then, because of Proposition 9, there exists a unique $\mathcal{N}[F, G, \lambda]=-i+O(\lambda)$ such that $\mathcal{M}[F, G, \lambda]=\mathcal{M}(\mathcal{N})$ on shell of (3.5). Hence (3.5) is equivalent to (3.1) and therefore gives well defined constitutive relations.

ii) If the constitutive relations (3.5) are a set of 6 independent relations that determine $G$ in terms of $F$ then the matrix entry $\mathcal{M}_{22} \neq 0$ (because otherwise from (3.5), we would have ${ }^{*} F=-\mathcal{M}_{21} F$ that constraints the independent fields $F$ ). It follows that (3.5) is equivalent to $G=-\mathcal{M}_{22}^{-1} \mathcal{M}_{21} F-\mathcal{M}_{22}^{-1 *} F$, i.e. to ${ }^{*} G=\mathcal{M}_{22}^{-1} F-\mathcal{M}_{22}^{-1} \mathcal{M}_{21}{ }^{*} F$. Repeating the argument that lead from (3.1) to (3.3) we conclude that (3.5) is equivalent 
to the equations

$$
\left(\begin{array}{c}
{ }^{*} F \\
{ }^{*} G
\end{array}\right)=\left(\begin{array}{cc}
0 & -1 \\
1 & 0
\end{array}\right)\left(\begin{array}{cc}
\mathcal{M}_{22}^{-1}+\mathcal{M}_{22}^{-1} \mathcal{M}_{21}^{2} & \mathcal{M}_{21} \\
\mathcal{M}_{21} & \mathcal{M}_{22}
\end{array}\right)\left(\begin{array}{l}
F \\
G
\end{array}\right) .
$$

We show that on shell of the relations (3.5) the matrix $\mathcal{M}[F, G, \lambda]$ is symmetric and symplectic because

$$
\mathcal{M}[F, G, \lambda]=\left(\begin{array}{cc}
\mathcal{M}_{22}^{-1}+\mathcal{M}_{22}^{-1} \mathcal{M}_{21}^{2} & \mathcal{M}_{21} \\
\mathcal{M}_{21} & M_{22}
\end{array}\right) \quad \text { (on shell). }
$$

Since by hypothesis the relations (3.5) determine $G$ in terms of $F$ and $^{*} G=-F+O(\lambda)$, we can also determine $F$ in terms of $G$ as a power series in $\lambda$. Then (3.5) is also equivalent to ${ }^{*} G=\mathcal{M}_{11} F+\mathcal{M}_{12} G$ and, observing that independence of the $G$ fields implies that the matrix entry $\mathcal{M}_{11} \neq 0$, we conclude that (3.5) is as well equivalent to $F=\mathcal{M}_{11}^{-1 *} G-\mathcal{M}_{11}^{-1} \mathcal{M}_{12} G$, that we rewrite as

$$
F^{+}=P G^{+}, \quad P \equiv\left(-\mathcal{M}_{11}^{-1}-i \mathcal{M}_{11}^{-1} \mathcal{M}_{12}\right) .
$$

Similarly (3.6) is also equivalent to its second row, ${ }^{*} G=\left(\mathcal{M}_{22}^{-1}+\mathcal{M}_{22}^{-1} \mathcal{M}_{21}^{2}\right) F+\mathcal{M}_{21} G$ that we rewrite as

$$
F^{+}=Q G^{+}, \quad Q \equiv\left(-\left(M_{22}^{-1}+M_{22}^{-1} M_{21}^{2}\right)^{-1}-i\left(M_{22}^{-1}+M_{22}^{-1} M_{21}^{2}\right)^{-1} M_{21}\right) .
$$

Independence of the fields $G^{+}$implies that subtracting (3.9) to (3.8) we obtain that $P-Q=0$ in each region of spacetime where $G^{+} \neq 0$. Moreover $P-Q=0$ in each region of spacetime where $G^{+}=0$ because $G^{+}=0$ in that region implies $P=1$ and $Q=1$ in that same region (we consider $\mathcal{M}[F, G, \lambda]$ to be a local functional of $F$ and $G$ ). This shows the on shell equality $P=Q$. Then equality (3.7) immediately follows.

Note 11. We have assumed that the constitutive relations can be written as power series expansions in $\lambda$. We here relax this assumption and consider constitutive relations (3.1) such that $\mathcal{N}[F, G, \lambda]=-i$ (or $\mathcal{M}[F, G, \lambda]=-1$ ) for the field configuration $F=G=0$; this is equivalent to state that for weak and slowly varying fields ${ }^{*} G \approx-F$ (i.e., that in this regime the constitutive relations are those of usual electromagnetism). Then applying the implicit function theorem to the constitutive relations (3.1) we know that there exists neighbourhoods of the field configurations $F=0, G=0$ such that (3.1) are equivalent to the explicit expressions $G=G[F, \lambda]$ and $F=F[G, \lambda]$. The result of this section therefore holds also without the power series expansion in $\lambda$ assumption: just consider fields sufficiently weak and slowly varying.

\subsection{Complex variables}

As in section 2.3 it is fruitful to consider the complex variables $T=F-i G, \bar{T}=F+i G$. The transition from the real to the complex variables is given by the symplectic and unitary matrix $\mathcal{A}^{t}$ where

$$
\mathcal{A}=\frac{1}{\sqrt{2}}\left(\begin{array}{cc}
1 & 1 \\
-i & i
\end{array}\right), \quad \mathcal{A}^{-1}=\mathcal{A}^{\dagger}
$$


The equation of motions in these variables read $d T=0$, with constitutive relations obtained applying the matrix $\mathcal{A}^{t}$ to (3.5):

$$
\left(\begin{array}{c}
{ }^{*} T \\
{ }^{*} \bar{T}
\end{array}\right)=-i\left(\begin{array}{cc}
1 & 0 \\
0 & -1
\end{array}\right) \mathcal{A}^{t} \mathcal{M} \overline{\mathcal{A}}\left(\begin{array}{c}
T \\
\bar{T}
\end{array}\right),
$$

where $\mathcal{A}^{t} \mathcal{M} \overline{\mathcal{A}}$, on shell of (3.11), is complex symplectic and pseudounitary w.r.t. the metric $\left(\begin{array}{cc}1 & 0 \\ 0 & -1\end{array}\right)$, i.e. it belongs to $\operatorname{Sp}(2, \mathbb{C}) \cap \mathrm{U}(1,1)=\mathrm{SU}(1,1)$. It is also Hermitian and negative definite. These properties uniquely characterize the matrices $\mathcal{A}^{t} \mathcal{M} \overline{\mathcal{A}}$ as the matrices

$$
\left(\begin{array}{cc}
-\sqrt{1+\tau \bar{\tau}} & -i \tau \\
i \bar{\tau} & -\sqrt{1+\tau \bar{\tau}}
\end{array}\right)
$$

where $\tau=\tau[T, \bar{T}]$ is a complex field that depends on $T, \bar{T}$ and possibly also their derivatives. We then see that the constitutive relations (3.11) are equivalent to the equations

$$
{ }^{*} T_{\mu \nu}=i \sqrt{1+\tau \bar{\tau}} T_{\mu \nu}-\tau \bar{T}_{\mu \nu}
$$

Notice that if $\mathcal{M}=-1+O(\lambda)$ (or equivalently $\mathcal{N}=-i+O(\lambda)$ ), then $\tau=O(\lambda)$. In particular electromagnetism is obtained setting $\tau=0$.

In conclusion equations (3.13) are the most general way of writing six independent real equations that allow to express $G=\frac{i}{2}(T+\bar{T})$ in terms of $F=\frac{1}{2}(T+\bar{T})$ as in $(3.1)$ (equivalently $F$ in terms of $G$ ). These constitutive relations are determined by a complex function $\mathcal{N}$ (depending on $F, G$ and their derivatives $\mathcal{N}=\mathcal{N}[F, G]$ ) or equivalently $\tau$ (depending on $T, \bar{T}$ and their derivatives $\tau=\tau[T, \bar{T}]$ ).

\section{Schrödinger approach to self-duality conditions}

In the previous section we have clarified the structure of the constitutive relations for an arbitrary nonlinear theory of electromagnetism. The theory can also be with higher derivatives of the field strength because the complex field $\mathcal{N}$, or equivalently the matrix $\mathcal{M}$ in (3.5) of (pseudo)scalar entries, can depend also on derivatives of the electric and magnetic field strengths $F$ and $G$.

We now further examine the constitutive relations for theories that satisfy the NGZ self-duality condition (2.24), i.e., $\bar{T} \widetilde{T}=0$, or equivalently,

$$
\bar{T}^{*} T=0 .
$$

The constitutive relations (3.13) determine the dependence of the magnetic field strength $G$ form the electric one $F$ or vice versa. We notice that this dependence is determined also if we constrain the fields in (3.13) to satisfy the condition $T^{*} T \neq 0$. This is so because the set of field configurations satisfying $T \widetilde{T} \neq 0$ is dense in the set of unconstrained field configurations. Hence if we multiply or divide the constitutive relations (3.13) by $T \widetilde{T}$ we obtain a set of equivalent constitutive relations. Having explained why we can freely divide by $T \widetilde{T}$ we can state the following 
Proposition 12. The constitutive relations (3.13) and the self-duality conditions (4.1) are equivalent to defining a nonlinear and higher derivatives extension of usual electromagnetism by the relations

$$
{ }^{*} T_{\mu \nu}=-\frac{T^{2}}{T^{*} T} T_{\mu \nu}-\tau \bar{T}_{\mu \nu},
$$

that henceforth we call self-dual constitutive relations in Schrödinger variables.

Equivalently we have the self-dual constitutive relations

$$
\begin{aligned}
{ }^{*} T_{\mu \nu} & =-\frac{T^{2}}{T^{*} T} T_{\mu \nu}-\frac{T \bar{T}}{\overline{T^{*} T}} \bar{T}_{\mu \nu}, \\
T \bar{T} & =r\left|T^{*} T\right|
\end{aligned}
$$

where the second equation is a scalar equation where $\left|T^{*} T\right|$ is the modulus of $T^{*} T$ and $r$ is a dimensionless scalar field that depends on $T, \bar{T}$ and their derivatives, that takes values in the non-negative real number and that is duality invariant.

Proof. Contracting the indices of $(3.13)$ with ${ }^{*} T_{\mu \nu}$ we obtain

$$
-T^{2}=i \sqrt{1+\tau \bar{\tau}} T^{*} T .
$$

Hence the self-duality condition (4.1) (i.e. (2.24)), and the constitutive relations (3.13) imply (4.2).

Vice versa (4.2) implies (4.1) and (3.13). Indeed, contracting (4.2) with ${ }^{*} T_{\mu \nu}$ we obtain $\bar{T}^{*} T=0$. This is trivially the case if $\tau \neq 0$. It holds also if $\tau=0$ because then (4.2) reads ${ }^{*} T=-\frac{T^{2}}{T^{*} T} T$, i.e., $\left(T^{*} T\right)^{2}=-T^{2} T^{2}$ that implies $T= \pm i^{*} T$, i.e., $F= \pm^{*} G$. This last relation implies the self-duality condition (4.1).

In order to show that (4.2) implies (3.13) first we contract (4.2) with ${ }^{*} \bar{T}_{\mu \nu}$, and obtain

$$
T \bar{T}=\tau \overline{T^{*} T} .
$$

Then we contract (4.2) with $T_{\mu \nu}$, and obtain

$$
T^{*} T=-\frac{T^{2}}{T^{*} T} T^{2}-\tau T \bar{T}
$$

This expression and the complex conjugate of (4.6) imply $1+\tau \bar{\tau}=-\frac{T^{2} T^{2}}{\left(T^{*} T\right)^{2}}$, and hence $-\frac{T^{2}}{T^{*} T}=i \sqrt{1+\tau \bar{\tau}}$, that substituted in (4.6) gives (3.13), as was to be proven. The sign of the square root $\sqrt{1+\tau \bar{\tau}}$ is determined considering the limit $\lambda \rightarrow 0$, where we want to recover usual electromagnetism, that in these variables reads ${ }^{*} T=i T$.

The self-duality condition $\bar{T}^{*} T=0$ implies (4.6) that fixes the phase of $\tau$ to equal that of $T^{*} T$. This constraint is automatically satisfied by setting $r=|\tau|$ and

$$
\tau=r \frac{T^{*} T}{\left|T^{*} T\right|} .
$$

The equivalence of (4.2) with the self-dual constitutive relations (4.3), (4.4) is then immediate. Trivially $r \geq 0$. Finally, recalling that $F$ and ${ }^{*} G$ are tensors while ${ }^{*} F$ and $G$ 
are pseudo-tensors we easily check that $T \bar{T}$ and $T^{*} T \overline{T^{*} T}$ are scalars, hence $r$ is a scalar field depending on $T, \bar{T}$ and their derivatives (i.e., $r$ is invariant under orientation reversal). Duality invariance of $r$ (under $T \rightarrow e^{-i \alpha} T$ ) immediately follows from (4.4).

In the self-duality conditions (4.3), (4.4) we have been able to disentangle the general relations that a self-dual theory must satisfy, i.e., (4.3), from the specific condition that defines the nonlinear theory: the scalar equation (4.4) that determines the ratio $T \bar{T} /\left|T^{*} T\right|$. Nonlinear self-dual theories are defined by imposing that this ratio equals an arbitrary duality invariant real and nonnegative scalar function $r$ of $T, \bar{T}$ and their derivatives.

Examples 13. Linear electromagnetism $\left(G={ }^{*} F\right)$ corresponds to the case $r=0$. Indeed $T \bar{T}=0$ in linear electromagnetism, while $T^{*} T=2 F^{*} F+2 i F^{2}$ is arbitrary.

Born-Infeld nonlinear theory satisfies the constitutive relations, $\lambda^{*} \bar{T}^{\mu \nu}=\frac{\partial}{\partial T_{\mu \nu}}\left(\frac{4 T^{2}}{T^{*} T}\right)$, i.e.,

$$
{ }^{*} T_{\mu \nu}=-\frac{T^{2}}{T^{*} T} T_{\mu \nu}-\frac{\lambda}{8}\left(T^{*} T\right) \bar{T}_{\mu \nu}
$$

as remarked by Schrödinger [1], see [34] for a clear account in nowadays notations. Comparison with (4.3) and (4.4), shows that Born-Infeld theory is determined by

$$
r=\frac{\lambda}{8}\left|T^{*} T\right| .
$$

We gain further insights in the self-dual constitutive relations by analyzing the phases and moduli of the scalars fields that enter (4.3) and (4.4). Relation (4.5) implies that the phase of $T^{*} T$ is bigger than the phase of $T^{2}$ by a $\pi / 2$ angle. In polar coordinates we have,

$$
T^{2}=\left|T^{2}\right| e^{i \varphi}, \quad T^{*} T=i\left|T^{*} T\right| e^{i \varphi} .
$$

Use of (4.6) leads to the relation $\tau \bar{\tau}=|T \bar{T}|^{2} /\left|T^{*} T\right|^{2}$, that inserted in (4.5) gives ${ }^{8}$

$$
\left|T^{2}\right|^{2}=\left|T^{*} T\right|^{2}+|T \bar{T}|^{2} .
$$

\subsection{Chiral variables}

The self-dual constitutive relations further simplify when we rewrite them in term of the chiral variables $T^{+}, T^{-}$and their complex conjugates.

We consider the Hodge dual of equation (4.3), sum it to $\pm i$-times (4.3), and, with the help of (2.28) and (2.29), we obtain the equivalent relations

$$
T_{\mu \nu}^{ \pm}=-\frac{T \bar{T}}{2 T^{\mp^{2}}} \frac{T^{*} T}{\bar{T}^{*} T} \bar{T}_{\mu \nu}^{\mp}
$$

where $2 T^{\mp^{2}}=T^{2} \mp i T^{*} T=\left(\left|T^{2}\right| \pm\left|T^{*} T\right|\right) e^{i \varphi}$. Further use of the phase relations (4.11) leads to $T_{\mu \nu}^{ \pm}=\frac{T \bar{T}}{2 \overline{T F}^{2}} \bar{T}_{\mu \nu}$, i.e., to

$$
T_{\mu \nu}^{+}=t e^{i \varphi} \overline{T^{-}}
$$

\footnotetext{
${ }^{8}$ This equation suggests to set $\cosh \beta=\rho_{T^{2}} / \rho_{T^{*} T}, \sinh \beta=\rho_{T \bar{T}} / \rho_{T^{*} T}$, so that (4.12) is automatically satisfied. With these variables the constitutive relations read ${ }^{*} T=i \cosh \beta T-i \sinh \beta \frac{T^{2}}{\rho_{T^{2}}} \bar{T}$. Different nonlinear theories are determined by the dependence of the angle $\beta$ from the fields $T, \bar{T}$ and their derivatives.
} 
and $T_{\mu \nu}^{-}=t^{-} e^{i \varphi} \overline{T^{+}}{ }_{\mu \nu}$, where the dimensionless, nonnegative and duality rotation invariant scalar fields $t$ and $t^{-}$are defined by

$$
t \equiv \frac{T \bar{T}}{\left|T^{2}\right|+\left|T^{*} T\right|}
$$

and $t^{-} \equiv \frac{T \bar{T}}{\left|T^{2}\right|-\left|T^{*} T\right|}$. Equations $T_{\mu \nu}^{-}=t^{-} e^{i \varphi} \overline{T^{+}}{ }_{\mu \nu}$ are equivalent to $T_{\mu \nu}^{+}=t e^{i \varphi} \overline{T^{-}}{ }_{\mu \nu}$ because, due to (4.12), $t^{-}=t^{-1}$.

The scalar equation (4.4) determines the value of the ratio $T \bar{T} /\left|T^{*} T\right|$. Because of the moduli relation (4.12), it equivalently determines the ratio $t$ in (4.15). Therefore, as in the previous section (see paragraph after the proof of Proposition 12), we can conclude that (4.14) is the general relation that a self-dual theory must satisfy, while the specific condition that defines the nonlinear theory is the dependence of the real nonnegative duality invariant scalar function $t$ from a set of independent variables and their derivatives, for example $T^{-}$and $\overline{T^{-}}$.

It is useful to present the explicit relation between the ratio $r=T \bar{T} /\left|T^{*} T\right|$ and $t$. We calculate

$$
\left|T^{-2}\right|\left(1-t^{2}\right)=\frac{1}{2}\left(\left|T^{2}\right|+\left|T^{*} T\right|\right)\left(1-t^{2}\right)=\left|T^{*} T\right|,
$$

multiply this last equality by $r=T \bar{T} /\left|T^{*} T\right|$ and obtain

$$
\left(1-t^{2}\right) r=2 t
$$

\section{Nonlinear theories without higher derivatives}

If the constitutive relations $G_{\mu \nu}=h_{\mu \nu}[F, \lambda]$ (see (2.3)) do not involve derivatives of the fields then, as noticed in the introduction, any antisymmetric 2-tensor is a linear combination of $F_{\mu \nu}$ and ${ }^{*} F_{\mu \nu}$ with coefficients that are (pseudo)scalar functions of $F_{\mu \nu}$. Hence the constitutive relations (3.1) or (3.3) are the most general ones. Furthermore, if we are in Minkowski spacetime Lorentz invariance implies that the field $\mathcal{N}$ in (3.1) and the matrix $\mathcal{M}$ in (3.3) can be expressed in terms of the Lorentz invariant combinations $F^{2}$ and $\left(F^{*} F\right)$. Similarly, if we choose the chiral fields $T^{-}$and $\overline{T^{-}}$as independent variables (cf. sections 2.4 and 4.1) then any Lorentz invariant field is a function of $T^{-2}$ and ${\overline{T^{-}}}^{2}$.

More in general we consider theories in curved spacetime that depend only on $F^{2}$ and $F^{*} F$, or $T^{-2}$ and ${\overline{T^{-}}}^{2}$. Since the action functional $\mathcal{I}\left[T^{-}, \overline{T^{-}}\right]$studied in section 2.4 and the scalar field $t$ defined in (4.15) are duality invariant, and under a duality of angle $\alpha$ we have the phase rotation $T^{-2} \rightarrow e^{2 i \alpha} T^{-2}$, we conclude that $\mathcal{I}$ and $t$ depend only on the modulus of $T^{-2}$, hence $\mathcal{I}=\mathcal{I}\left[T^{-}, \overline{T^{-}}\right]$and $t=t\left[T^{-}, \overline{T^{-}}\right]$simplify to

$$
\mathcal{I}=\frac{1}{\lambda} \int d^{4} x \sqrt{g} I(u), \quad t=t(u),
$$

where $I(u)$ is an adimensional scalar function, and the variable $u$ is defined by

$$
u \equiv 2 \lambda\left|T^{-2}\right|=\lambda\left(\left|T^{2}\right|+\left|T^{*} T\right|\right)
$$


Similarly, the constitutive relations (2.32) simplify to

$$
T^{+\mu \nu}=\frac{1}{\lambda} \frac{\partial I}{\partial \overline{T^{-}}{ }_{\mu \nu}}=\frac{1}{\lambda} \frac{d I}{d u} \frac{\partial u}{\partial \overline{T^{-}}{ }_{\mu \nu}},
$$

and comparison with with (4.14) leads to

$$
t=2 \frac{d I}{d u} .
$$

In fact, deriving $u^{2}$ we obtain $\frac{\partial u}{\partial \overline{\bar{T}^{-}} \nu}=2 \lambda e^{i \varphi} \overline{T^{-}}{ }_{\mu}$ where we used the same conventions as in footnote 3 , and that $T^{-2}=\left|T^{-2}\right| e^{i \varphi}$ (see expression immediately after (4.13)).

\subsection{Born-Infeld nonlinear theory}

In this section we determine the scalar field $t=t(u)=2 \frac{d I}{d u}$ in case of Born-Infeld theory. This is doable thanks to Schrödinger's formulation (4.9) of Born-Infeld theory, that explicitly gives $r=\frac{\lambda}{8}\left|T^{*} T\right|$, see (4.10). Then from (4.16) we have

$$
r=\frac{1}{16} u\left(1-t^{2}\right),
$$

and recalling (4.17) we obtain $[37,33]$

$$
\left(1-t^{2}\right)^{2} u=32 t
$$

Now in the limit $u \rightarrow 0$, i.e., $\lambda \rightarrow 0$, we see from (4.15) that $t \rightarrow 0$. The function $t=t(u)$ defining Born-Infeld theory is therefore given by the unique positive root of the fourth order polynomial equation (5.6) that has the correct $\lambda \rightarrow 0$ limit. Explicitly,

$$
t=\frac{1}{\sqrt{3}}\left(\sqrt{1+s+s^{-1}}-\sqrt{2-s-s^{-1}+\frac{24 \sqrt{3}}{u \sqrt{1+s+s^{-1}}}}\right),
$$

where

$$
s=\frac{1}{u}\left(216 u+12 \sqrt{3} \sqrt{108+u^{2}} u+u^{3}\right)^{\frac{1}{3}}
$$

\subsection{The hypergeometric function and its hidden identity}

In [26] the action functional $\mathcal{I}$ and the function $t(u)$ corresponding to the Born-Infeld action were found via an iterative procedure order by order in $\lambda$ (or equivalently in $u$ ). The first coefficients of the power series expansion of $t(u)$ were recognized to be those of a generalized hypergeometric function, leading to the conclusion

$$
t(u)=\frac{u}{32}{ }_{3} F_{2}\left(\frac{1}{2}, \frac{3}{4}, \frac{5}{4} ; \frac{4}{3}, \frac{5}{3} ;-\frac{u^{2}}{3^{3} \cdot 2^{2}}\right),
$$

and, integrating (5.4),

$$
I(u)=6\left(1-{ }_{3} F_{2}\left(-\frac{1}{2},-\frac{1}{4}, \frac{1}{4}, \frac{1}{3}, \frac{2}{3} ;-\frac{u^{2}}{3^{3} \cdot 2^{2}}\right)\right) .
$$


We have checked that the expansion in power series of $u$ of the closed form expression of $t(u)$ derived in (5.7), (5.8) coincides, up to order $O\left(u^{1000}\right)$ with $\frac{u}{32}$ times the hypergeometric function in (5.9). Therefore we conjecture that the hypergeometric function in (5.9)

$$
\mathfrak{F}\left(u^{2}\right) \equiv{ }_{3} F_{2}\left(\frac{1}{2}, \frac{3}{4}, \frac{5}{4} ; \frac{4}{3}, \frac{5}{3} ;-\frac{u^{2}}{3^{3} \cdot 2^{2}}\right)=2 \sum_{k=0}^{\infty} \frac{(4 k+1) !}{(3 k+2) ! k !}\left(-\frac{u^{2}}{4^{5}}\right)^{k}
$$

has the closed form expression $\mathfrak{F}\left(u^{2}\right)=\frac{32}{u} t(u)$ where $t(u)$ is given in (5.7), (5.8), and, because of (5.6), that it satisfies the "hidden" identity

$$
\mathfrak{F}\left(u^{2}\right)=\left(1-\frac{u^{2}}{4^{5}} \mathfrak{F}\left(u^{2}\right)^{2}\right)^{2} .
$$

It is indeed this identity that we have verified up to $O\left(u^{1000}\right)$.

\subsection{General nonlinear theory}

Since Born-Infeld theory is singled out by setting $r=\frac{\lambda}{8}\left|T^{*} T\right|$, and Maxwell theory by setting $r=0$ (cf. Example 13), it is convenient to describe a general nonlinear theory without higher derivatives by setting

$$
r=\frac{\lambda}{8}\left|T^{*} T\right| f(u) / u
$$

where $f(u)$ is a positive function of $u$. We require the theory to reduce to electromagnetism in the weak field limit, i.e., ${ }^{*} G_{\mu \nu}=-F+o(F)$ for $F \rightarrow 0$. Then we have $T^{-}=\mathcal{O}(F), T^{+}=$ $o(F), u=\mathcal{O}\left(F^{2}\right)$. Hence from (4.14) we obtain $\lim _{u \rightarrow 0} t=0$. Moreover from (4.17), $r=$ $\mathcal{O}(t)$ and from $r=\frac{1}{16} f(u)\left(1-t^{2}\right)$ (that follows from (5.13) and (4.16)) $f=\mathcal{O}(t)$. Hence the theory reduces to electromagnetism in the weak field limit if and only if $\lim _{u \rightarrow 0} f(u)=0 .{ }^{9}$

From $r=\frac{1}{16} f(u)\left(1-t^{2}\right)$ (that follows from (5.13) and (4.16)) and (4.17) we obtain that the composite function $t(f(u))$ satisfies the fourth order polynomial equation

$$
\left(1-t^{2}\right)^{2} f(u)=32 t
$$

so that $t(f(u))$ is obtained with the substitution $u \rightarrow f(u)$ in (5.7) and (5.8), or in (5.9).

More explicitly, recalling the constitutive relation (4.2), we conclude that the constitutive relations à la Schrödinger

$$
{ }^{*} T_{\mu \nu}=-\frac{T^{2}}{T^{*} T} T_{\mu \nu}-\frac{\lambda}{8} \frac{f(u)}{u}\left(T^{*} T\right) \bar{T}_{\mu \nu}
$$

\footnotetext{
${ }^{9}$ We further notice that $\lim _{u \rightarrow 0} f(u)=0$ implies $I(u)=o(u)$. In particular the theory defined by $I(u)=u$ (or equivalently $f(u)=\frac{2^{6}}{3^{2}}$ ) does not reduce to electromagnetism for weak fields.

In general, besides requiring that the theory determined by $f(u)$ reduces to electromagnetism in the weak field limit we can also require the theory to be analytic in $F$ (the lagrangian to have a power series expansion in $F$ around $F=0)$. In this case from (2.40) and inverting relations (2.42), or more explicitly from (2.39), we see that the Legendre transformed function $I(u)$ must depend on $u^{2}=4 \lambda^{2} T^{-2}{\overline{T^{-}}}^{2}$. Equivalently $f(u) / u$ must depend on $u^{2}$.
} 
are equivalent to the constitutive relations (deformed twisted self-duality conditions)

$$
T^{+\mu \nu}=\frac{1}{2 \lambda} t(f(u)) \frac{\partial u}{\partial \overline{T^{-}}{ }^{\prime} \nu},
$$

where $t(f(u))$ satisfies the quartic equation (5.14), and we recall that $u=2 \lambda\left|T^{-2}\right|=$ $\lambda\left(\left|T^{2}\right|+\left|T^{*} T\right|\right)$.

In other words the appearence of the quartic equation (5.14) is a general feature of the relation between the constitutive relations (5.15) and (5.16), it appears for any self-dual theory and it is not only a feature of the Born-Infeld theory.

\section{Acknowledgments}

We thank W. Chemissany, R. Kallosh, T. Ortin and M. Trigiante for valuable correspondence during last fall. In particular we thank R. Kallosh for discussions and her interest on the relation between the hypergeometric function of her work [26] and the quartic equation expressing Born Infeld theory in duality invariant variables. We also thank F. Bonechi for fruitful discussions.

The hospitality of CERN Theory Unit where the present work has been initiated is also gratefully acknowledged. This work is supported by the ERC Advanced Grant no. 226455, Supersymmetry, Quantum Gravity and Gauge Fields (SUPERFIELDS).

\section{A Examples of higher derivatives theories}

We construct examples of higher derivatives U(1) actions that define self-dual theories. These examples include the Bossard Nicolai one in [25]; the actions we present are quadratic in the field strength. Let

$$
S=-\frac{1}{4} \int d^{4} x \sqrt{g} F O F
$$

with $O$ a matrix $O_{\mu \nu}^{\rho \sigma}$ of differential operators independent from $F$; explicitly $F O F=$ $F^{\mu \nu} O_{\mu \nu}^{\rho \sigma} F_{\rho \sigma}$. We recall that by definition the hermitian conjugate operator $O^{\dagger}$ satisfies $\int\left(O^{\dagger} K\right) F=\int K O F$ for all antisymmetric and real tensors $K$ and $F$. Since $\int F O F=$ $\int\left(O^{\dagger} F\right) F=\int F\left(O^{\dagger} F\right)$, there is no restriction in considering $O$ hermitian, i.e., $\int(O K) F=$ $\int K O F$, or explicitly $\int d^{4} x \sqrt{g}\left(O_{\rho \sigma}^{\mu \nu} K_{\mu \nu}\right) F^{\rho \sigma}=\int d^{4} x \sqrt{g} K^{\mu \nu} O_{\mu \nu}^{\rho \sigma} F_{\rho \sigma}$. Let $O$ also satisfy

$$
O \circ * \circ O=*
$$

i.e., $O^{*}(O F)={ }^{*} F$.

We show that the action (A.1) gives self-dual equations of motion if $O$ satisfies (A.2). Indeed in this case the self-duality condition (2.23), i.e., $\int d^{4} x F \widetilde{F}+G \widetilde{G}=0$, holds. The proof is easy. We first calculate

$$
\widetilde{G}^{\mu \nu}=2 \frac{\delta S}{\delta F_{\mu \nu}}=-\sqrt{g} O^{\mu \nu \rho \sigma} F_{\rho \sigma},
$$


i.e., ${ }^{*} G^{\mu \nu}=-(O F)^{\mu \nu}$. Hence

$$
\begin{aligned}
\int d^{4} x \widetilde{G} G & =\int d^{4} x \sqrt{g}\left({ }^{*} G\right) G=-\int d^{4} x \sqrt{g}\left({ }^{*} G\right){ }^{*}\left({ }^{*} G\right) \\
& =-\int d^{4} x \sqrt{g}(O F){ }^{*}(O F)=-\int d^{4} x \sqrt{g} F O^{*}(O F) \\
& =-\int d^{4} x \sqrt{g} F^{*} F=-\int d^{4} x F \widetilde{F},
\end{aligned}
$$

where in the fourth equality we used (A.2).

Examples of differential operators $O$ are given by considering operators $\Delta$ on antisymmetric tensors $F_{\mu \nu}$ that satisfy the hermiticity condition $\Delta^{\dagger}=\Delta$ and that anticommute with the $*$-Hodge operator,

$$
* \circ \Delta=-\Delta \circ^{*} \text {. }
$$

Let's introduce a coupling constant $\lambda$ so that $\lambda \Delta$ is adimensional, and let $f(\lambda \Delta)$ be an odd function in $\Delta$ (e.g., a polynomial, or a power series function like $\lambda \Delta, \lambda \Delta^{3}, \sin (\lambda \Delta)$ ). Then $* \circ f(\lambda \Delta)=-f(\lambda \Delta) \circ *$, and

$$
O=(1-f(\lambda \Delta))^{-1}(1+f(\lambda \Delta))
$$

satisfies (A.2).

In particular, if $f(\lambda \Delta)=\lambda \Delta$ and if $\Delta_{\mu \nu}^{\rho \sigma} F_{\rho \sigma}=\nabla_{\kappa}\left(T_{[\mu}{ }^{\kappa \lambda[\sigma} \nabla_{\lambda} \delta_{\nu]}^{\rho]} F_{\rho \sigma}\right)$, where the covariant derivatives are with respect to the Levi-Civita connection, $T^{\mu \kappa \lambda \sigma}$ is the Bel-Robinson tensor, and the square brackets denote antisymmetrization in the embraced indices, then we obtain the action of Bossard and Nicolai [25].

\section{B The action functional $S[F]$ from $\mathcal{I}\left[T^{-}, \overline{T^{-}}\right]$}

We here determine the first two nontrivial terms $S^{(1)}$ and $S^{(2)}$ of the action $S$, see $(2.39)$ section 2.4.

Since $S^{(0)}=-\frac{1}{4} \int d^{4} x \sqrt{g} F^{2}$ corresponds to $\mathcal{I}^{[0]}=0$, we have (cf. $\left.(2.38)\right), T^{+(0)}=0$, $T^{-(0)}=2 F^{-}, G^{-(0)}=i F^{-}$, and, for $n \geq 1, T^{-(n)}=-i G^{-(n)},{\overline{T^{-}}}^{(n)}=i G^{+(n)}$. The following useful formula is then easily derived using the chain rule:

$$
\frac{\left.\delta \mathcal{I}^{[m]}\right|_{F^{\mp}} ^{(n)}}{\delta F^{-}}=\left.2 \frac{\delta \mathcal{I}^{[m]}}{\delta T^{-}}\right|_{F^{\mp}} ^{(n)}-\left.2 \sum_{p=m}^{n-1} \int d^{4} x \frac{1}{\sqrt{g}} \frac{\delta \mathcal{I}^{[m]}}{\delta T^{-}}\right|_{F^{\mp}} ^{(p)} \frac{\delta^{2} S^{(n-p)}}{\delta F^{-} \delta F^{-}}+\left.\frac{\delta \mathcal{I}^{[m]}}{\delta \overline{T^{-}}}\right|_{F^{\mp}} ^{(p)} \frac{\delta^{2} S^{(n-p)}}{\delta F^{-} \delta F^{+}}
$$

where we have simplified the notation by setting $\left.\right|_{F^{\mp}}=\left.\right|_{\frac{T^{-}\left[F^{-}, F^{+}\right]}{T^{-}\left[F^{-}, F^{+}\right]}}$and omitting spacetime indices, and where we have assumed that we know the action $S[F]$ up to order $n-1$ so that, for all $p=1,2, \ldots n-1$, we have $\mp i G^{ \pm(p)}=\frac{2}{\sqrt{g}} \frac{\delta^{2} S^{(p)}}{\partial F^{ \pm}}$, and therefore $\frac{\delta T^{-(p)}}{\delta F^{-}}=-i \frac{\delta G^{-(p)}}{\delta F^{-}}=-\frac{2}{\sqrt{g}} \frac{\delta^{2} S^{(p)}}{\partial F^{-} \partial F^{-}}$.

If $m=n$ then the above formula simply reads $\frac{\left.\delta \mathcal{I}^{[n]}\right|_{F^{\mp}} ^{(n)}}{\delta F^{-}}=\left.2 \frac{\delta \mathcal{I}^{[n]}}{\delta T^{-}}\right|_{F^{\mp}} ^{(n)}$, and since $\left.\mathcal{I}^{[n]}\right|_{F^{\mp}} ^{(n)}=\mathcal{I}^{[n]}\left[2 F^{-}, 2 F^{+}\right]\left(\right.$use $\left.T^{-(0)}=2 F^{-}\right)$, it simplifies to

$$
\frac{\delta \mathcal{I}^{[n]}\left[2 F^{-}, 2 F^{+}\right]}{\delta F^{-}}=\left.2 \frac{\delta \mathcal{I}^{[n]}}{\delta T^{-}}\right|_{F^{\mp}} ^{(n)}
$$


Setting $n=1$ and recalling that since $\mathcal{I}^{[0]}=0,\left.\frac{\delta \mathcal{I}^{[1]}}{\delta T^{-}}\right|_{F^{\mp}} ^{(1)}=\left.\frac{\delta \mathcal{I}}{\delta T^{-}}\right|_{F^{\mp}} ^{(1)}$, we immediately see that $S^{(1)}\left[F^{-}, F^{+}\right]=\frac{1}{4} \mathcal{I}^{[1]}\left[2 F^{-}, 2 F^{+}\right]$satisfies $(2.36)$.

In order to determine $S^{(2)}$ we first calculate (using for example the chain rule in deriving w.r.t. $\lambda)$

$$
\begin{aligned}
\left.\mathcal{I}^{[1]}\right|_{F^{\mp}} ^{(2)} & =\left.\int d^{4} x \frac{\delta \mathcal{I}^{[1]}}{\delta T^{-}}\right|_{F^{\mp}} ^{(1)} T^{-(1)}+\left.\frac{\delta \mathcal{I}^{[1]}}{\delta \overline{T^{-}}}\right|_{F^{\mp}} ^{(1)}{\overline{T^{-}}}^{(1)} \\
& =2 \int d^{4} x \frac{\delta S^{(1)}}{\delta F^{-}}\left(-i G^{-}\right)^{(1)}+\frac{\delta S^{(1)}}{\delta F^{+}}\left(i G^{+}\right)^{(1)} \\
& =-4 \int d^{4} x \frac{1}{\sqrt{g}} \frac{\delta S^{(1)}}{\delta F^{-}} \frac{\delta S^{(1)}}{\delta F^{-}}+\frac{\delta S^{(1)}}{\delta F^{+}} \frac{\delta S^{(1)}}{\delta F^{+}}
\end{aligned}
$$

where in the second line we used $\left.\frac{\delta \mathcal{I}^{[1]}}{\delta T^{-}}\right|_{F^{\mp}} ^{(1)}=\left.\frac{\delta \mathcal{I}}{\delta T^{-}}\right|_{F^{\mp}} ^{(1)}$ and then $(2.36)$ at order $n=1$. In the third line we used the constitutive relations (2.20), i.e., $G^{-}=-\frac{2 i}{\sqrt{g}} \frac{\delta S}{\delta F^{-}}$at order $n=1$, that we already know to be implied by the chiral constitutive relations (2.32).

Next for notational simplicity we set $\int=\int d^{4} x \frac{1}{\sqrt{g}}$ and we compute

$$
\begin{aligned}
\left.\frac{\delta \mathcal{I}}{\delta T^{-}}\right|_{F^{\mp}} ^{(2)} & =\left.\frac{\delta \mathcal{I}^{[2]}}{\delta T^{-}}\right|_{F^{\mp}} ^{(2)}+\left.\frac{\delta \mathcal{I}^{[1]}}{\delta T^{-}}\right|_{F^{\mp}} ^{(2)} \\
& =\frac{1}{2} \frac{\mathcal{I}^{[2]}\left[2 F^{-}, 2 F^{+}\right]}{\delta F^{-}}+\frac{1}{2} \frac{\left.\delta \mathcal{I}^{[1]}\right|_{F^{\mp}} ^{(2)}}{\delta F^{-}}+\left.\int \frac{\delta \mathcal{I}^{[1]}}{\delta T^{-}}\right|_{F^{\mp}} ^{(1)} \frac{\delta^{2} S^{(1)}}{\delta F^{-} \delta F^{-}}+\left.\frac{\delta \mathcal{I}^{[1]}}{\delta \bar{T}^{-}}\right|_{F^{\mp}} ^{(1)} \frac{\delta^{2} S^{(1)}}{\delta F^{-} \delta F^{+}} \\
& =\frac{1}{2} \frac{\mathcal{I}^{[2]}\left[2 F^{-}, 2 F^{+}\right]}{\delta F^{-}}+\frac{1}{2} \frac{\left.\delta \mathcal{I}^{[1]}\right|_{F^{\mp}} ^{(2)}}{\delta F^{-}}+\frac{\delta}{\delta F^{-}} \int \frac{\delta S^{(1)}}{\delta F^{-}} \frac{\delta S^{(1)}}{\delta F^{-}}+\frac{\delta S^{(1)}}{\delta F^{+}} \frac{\delta S^{(1)}}{\delta F^{+}} \\
& =\frac{\delta}{\delta F^{-}}\left(\frac{1}{2} \mathcal{I}^{[2]}\left[2 F^{-}, 2 F^{+}\right]-\int \frac{\delta S^{(1)}}{\delta F^{-}} \frac{\delta S^{(1)}}{\delta F^{-}}+\frac{\delta S^{(1)}}{\delta F^{+}} \frac{\delta S^{(1)}}{\delta F^{+}}\right)
\end{aligned}
$$

where in the second line we have used (B.2) and (B.1), in the third line we have noticed again that $\left.\frac{\delta \mathcal{I}^{[1]}}{\delta T^{-}}\right|_{F^{\mp}} ^{(1)}=\left.\frac{\delta \mathcal{I}}{\delta T^{-}}\right|_{F^{\mp}} ^{(1)}=2 \frac{\delta S^{(1)}}{\delta F^{-}}$(cf. (2.36), in the fourth line we have used (B.3). From the equality (B.4) we see that $S^{(2)}=\frac{1}{4} \mathcal{I}^{[2]}\left[2 F^{-}, 2 F^{+}\right]-\frac{1}{2} \int \frac{\delta S^{(1)}}{\delta F^{-}} \frac{\delta S^{(1)}}{\delta F^{-}}+\frac{\delta S^{(1)}}{\delta F^{+}} \frac{\delta S^{(1)}}{\delta F^{+}}$ satisfies (2.36) with $n=2$.

\section{The energy momentum tensor and its trace}

We first recall that the symmetric energy-momentum tensor $\theta^{\mu \nu}$ of a nonlinear electromagnetic theory is given by

$$
\theta^{\mu \nu}=-\widetilde{G}^{\mu \lambda} F_{\lambda}^{\nu}+g^{\mu \nu} \mathcal{L}
$$

if the Lagrangian $L$ in the action $S[F]=\int d^{4} x \mathcal{L}=\frac{1}{\lambda} \int d^{4} x \sqrt{g} L$ depends on the field strength $F_{\mu \nu}$ and the metric $g_{\mu \nu}$ only via the invariant and dimensionless combinations

$$
\alpha=\frac{\lambda}{4} F^{2}, \quad \beta=\frac{\lambda}{4} F^{*} F .
$$


Indeed we compute

$$
\frac{\partial \alpha}{\partial g_{\mu \nu}}=-2 \frac{\partial \alpha}{\partial F_{\mu \rho}} F_{\rho}^{\nu}, \quad \frac{\partial \beta}{\partial g_{\mu \nu}}=-2 \frac{\partial \beta}{\partial F_{\mu \rho}} F_{\rho}^{\nu},
$$

(where the factor 2 is due to our $\frac{\partial}{\partial F_{\mu \rho}}$ conventions, cf. (2.20) and its footnote); for the second equation we used $\frac{\partial \sqrt{g}^{-1}}{\partial g_{\mu \nu}}=-\sqrt{g}^{-1} g^{\mu \nu}$, and the property ${ }^{*} F^{\mu \lambda} F_{\nu \lambda}=-\frac{1}{4} \delta^{\mu}{ }_{\nu}{ }^{*} F^{\rho \sigma} F_{\rho \sigma}$. Expression (C.1) for the energy momentum tensor $\theta^{\mu \nu}=\frac{\delta S}{\delta g_{\mu \nu}}$ is then straightforward.

Now an action in Minkowski spacetime that has no derivatives of the field strength $F$, by Lorentz invariance depends on $F$ only via the (pseudo)scalars $F^{2}$ and $F \widetilde{F}$. We can then always minimally couple the action to gravity so that the metric enters only in (C.2), and hence so that (C.1) holds. Even if the coupling to gravity (for example in order to preserve symmetry properties) requires terms like $R F^{2}$ where $R$ is the scalar curvature, expression (C.1) still holds in flat spacetime.

From (C.1) it follows that the trace of the energy momentum tensor satisfies

$$
\frac{1}{4} \theta^{\mu}{ }_{\mu}=\mathcal{L}-\frac{1}{4} \widetilde{G} F
$$

We therefore have

$$
\frac{1}{4} \int d^{4} x g_{\mu \nu} \frac{\delta S}{\delta g_{\mu \nu}}=\int d^{4} x \frac{1}{4} \theta_{\mu}^{\mu}=S-\frac{1}{4} \int d^{4} x \widetilde{G} F=-\lambda \frac{\partial S}{\partial \lambda},
$$

the last relation follows observing that the inverse metric $g^{\mu \nu}$ appears always with the factor $\lambda^{1 / 2}$ in the action $S[F]=\int d^{4} x \mathcal{L}=\frac{1}{\lambda} \int d^{4} x \sqrt{g} L$ (cf. (C.2)).

Finally if we let $S[F] \rightarrow S_{c}[F]=\frac{1}{c^{2}} S[c F]$, we see that (C.5) coincides with (2.13). Indeed $\lambda \frac{\partial}{\partial \lambda}$ equals $c^{2} \frac{\partial}{\partial c^{2}}$ because $S_{c}[F]$ depends only on the product $c^{2} \lambda$.

Open Access. This article is distributed under the terms of the Creative Commons Attribution License which permits any use, distribution and reproduction in any medium, provided the original author(s) and source are credited.

\section{References}

[1] E. Schrödinger, Contributions to Born's new theory of the electromagnetic field, Proc. Roy. Soc. London A 150 (1935) 465.

[2] M. Born and L. Infeld, Foundations of the new field theory, Proc. Roy. Soc. London A 144 (1934) 425.

[3] S. Ferrara, J. Scherk and B. Zumino, Algebraic properties of extended supergravity theories, Nucl. Phys. B 121 (1977) 393 [inSPIRE].

[4] E. Cremmer, J. Scherk and S. Ferrara, SU(4) invariant supergravity theory, Phys. Lett. B 74 (1978) 61 [INSPIRE].

[5] E. Cremmer and B. Julia, The SO(8) supergravity, Nucl. Phys. B 159 (1979) 141 [InSPIRE].

[6] E. Cremmer and B. Julia, The $N=8$ supergravity theory. 1. The lagrangian, Phys. Lett. B 80 (1978) 48 [INSPIRE]. 
[7] M.K. Gaillard and B. Zumino, Duality rotations for interacting fields, Nucl. Phys. B 193 (1981) 221 [inSPIRE].

[8] G.W. Gibbons and D.A. Rasheed, SL $(2, \mathbb{R})$ invariance of nonlinear electrodynamics coupled to an axion and a dilaton, Phys. Lett. B 365 (1996) 46 [hep-th/9509141] [INSPIRE].

[9] S. Deser and R. Puzalowski, Supersymmetric nonpolynomial vector multiplets and causal propagation, J. Phys. A 13 (1980) 2501 [INSPIRE].

[10] S. Cecotti and S. Ferrara, Supersymmetric Born-Infeld lagrangians, Phys. Lett. B 187 (1987) 335 [inSPIRE].

[11] J. Bagger and A. Galperin, A new goldstone multiplet for partially broken supersymmetry, Phys. Rev. D 55 (1997) 1091 [hep-th/9608177] [INSPIRE].

[12] S.V. Ketov, A manifestly $N=2$ supersymmetric Born-Infeld action, Mod. Phys. Lett. A 14 (1999) 501 [hep-th/9809121] [InSPIRE].

[13] S.M. Kuzenko and S. Theisen, Supersymmetric duality rotations, JHEP 03 (2000) 034 [hep-th/0001068] [INSPIRE].

[14] S.M. Kuzenko and S. Theisen, Nonlinear selfduality and supersymmetry, Fortsch. Phys. 49 (2001) 273 [hep-th/0007231] [INSPIRE].

[15] S. Bellucci, E. Ivanov and S. Krivonos, Towards the complete $N=2$ superfield Born-Infeld action with partially broken $N=4$ supersymmetry, Phys. Rev. D 64 (2001) 025014 [hep-th/0101195] [INSPIRE].

[16] M. Roček and A.A. Tseytlin, Partial breaking of global D $=4$ supersymmetry, constrained superfields and three-brane actions, Phys. Rev. D 59 (1999) 106001 [hep-th/9811232] [INSPIRE].

[17] S.V. Ketov, Born-Infeld-Goldstone superfield actions for gauge fixed D5 branes and D3 branes in 6D, Nucl. Phys. B 553 (1999) 250 [hep-th/9812051] [INSPIRE].

[18] Z. Bern, J. Carrasco, L.J. Dixon, H. Johansson and R. Roiban, Manifest ultraviolet behavior for the three-loop four-point amplitude of $N=8$ supergravity, Phys. Rev. D 78 (2008) 105019 [arXiv:0808.4112] [INSPIRE].

[19] J. Broedel and L.J. Dixon, $R^{4}$ counterterm and $E_{7(7)}$ symmetry in maximal supergravity, JHEP 05 (2010) 003 [arXiv:0911.5704] [INSPIRE].

[20] H. Elvang and M. Kiermaier, Stringy KLT relations, global symmetries and $E_{7(7)}$ violation, JHEP 10 (2010) 108 [arXiv: 1007.4813] [INSPIRE].

[21] N. Beisert et al., $E_{7(7)}$ constraints on counterterms in $N=8$ supergravity, Phys. Lett. B 694 (2010) 265 [arXiv: 1009.1643] [INSPIRE].

[22] G. Bossard, P. Howe and K. Stelle, On duality symmetries of supergravity invariants, JHEP 01 (2011) 020 [arXiv: 1009.0743] [INSPIRE].

[23] R. Kallosh, $E_{7(7)}$ symmetry and finiteness of $N=8$ supergravity, JHEP 03 (2012) 083 [arXiv:1103.4115] [INSPIRE].

[24] R. Kallosh, $N=8$ counterterms and $E_{7(7)}$ current conservation, JHEP 06 (2011) 073 [arXiv: 1104.5480] [INSPIRE].

[25] G. Bossard and H. Nicolai, Counterterms vs. dualities, JHEP 08 (2011) 074 [arXiv:1105.1273] [INSPIRE]. 
[26] J.J.M. Carrasco, R. Kallosh and R. Roiban, Covariant procedures for perturbative non-linear deformation of duality-invariant theories, Phys. Rev. D 85 (2012) 025007 [arXiv: 1108.4390] [INSPIRE].

[27] W. Chemissany, R. Kallosh and T. Ortín, Born-Infeld with higher derivatives, Phys. Rev. D 85 (2012) 046002 [arXiv:1112.0332] [INSPIRE].

[28] J. Broedel, J.J.M. Carrasco, S. Ferrara, R. Kallosh and R. Roiban, $N=2$ supersymmetry and U(1)-duality, Phys. Rev. D 85 (2012) 125036 [arXiv: 1202.0014] [INSPIRE].

[29] S.M. Kuzenko, Nonlinear self-duality in $N=2$ supergravity, JHEP 06 (2012) 012 [arXiv: 1202.0126] [INSPIRE].

[30] S.M. Kuzenko, Duality rotations in supersymmetric nonlinear electrodynamics revisited, JHEP 03 (2013) 153 [arXiv: 1301.5194] [INSPIRE].

[31] E.A. Ivanov and B.M. Zupnik, $N=3$ supersymmetric Born-Infeld theory, Nucl. Phys. B 618 (2001) 3 [hep-th/0110074] [INSPIRE].

[32] E.A. Ivanov and B.M. Zupnik, New approach to nonlinear electrodynamics: dualities as symmetries of interaction, Phys. Atom. Nucl. 67 (2004) 2188 [hep-th/0303192] [INSPIRE].

[33] E.A. Ivanov and B.M. Zupnik, Bispinor auxiliary fields in duality-invariant electrodynamics revisited, arXiv:1212.6637 [INSPIRE].

[34] M.K. Gaillard and B. Zumino, Nonlinear electromagnetic selfduality and Legendre transformations, hep-th/9712103 [INSPIRE].

[35] P. Aschieri, S. Ferrara and B. Zumino, Duality Rotations in Nonlinear Electrodynamics and in Extended Supergravity, Riv. Nuovo Cim. 31 (2008) 625 [arXiv:0807.4039] [INSPIRE].

[36] M. Hatsuda, K. Kamimura and S. Sekiya, Electric magnetic duality invariant Lagrangians, Nucl. Phys. B 561 (1999) 341 [hep-th/9906103] [INSPIRE].

[37] P. Aschieri and S. Ferrara, unpublished (Oct. 2012). 